\title{
Single-cell transcriptional dynamics and origins of neuronal diversity in the developing mouse neocortex
}

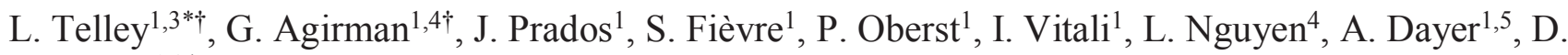 \\ Jabaudon ${ }^{1,2^{*}}$
}

${ }^{1}$ Department of Basic Neurosciences, University of Geneva, Geneva, Switzerland.

${ }^{2}$ Clinic of Neurology, Geneva University Hospital, Geneva, Switzerland.

${ }^{3}$ Current address: Department of Basic Neuroscience, University of Lausanne, Switzerland.

${ }^{4}$ GIGA-Neurosciences, University of Liège, C.H.U. Sart Tilman, Liège, Belgium.

${ }^{5}$ Department of Psychiatry, Geneva University Hospital, Geneva, Switzerland.

$\dagger$ equally contributed to this work.

*Correspondence to: ludovic.telley@unil.ch; denis.jabaudon@unige.ch

During cortical development, distinct subtypes of glutamatergic neurons are sequentially born and differentiate from dynamic populations of progenitors. The neurogenic competence of these progenitors progresses as corticogenesis proceeds; likewise, newborn neurons transit through sequential states as they differentiate. Here, we trace the developmental transcriptional trajectories of successive generations of apical progenitors (APs) and isochronic cohorts of their daughter neurons using parallel single-cell RNA sequencing between embryonic day (E) 12 and E15 in the mouse cerebral cortex. Our results identify the birthdate- and differentiation stagerelated transcriptional dynamics at play during corticogenesis. As corticogenesis proceeds, APs transit through embryonic age-dependent molecular states, which are transmitted to their progeny to generate successive initial daughter cell identities. In neurons, essentially conserved post-mitotic differentiation programs are applied onto these distinct AP-derived ground states, allowing temporally-regulated sequential emergence of specialized neuronal cell types. Molecular temporal patterning of sequentially-born daughter neurons by their respective mother cell thus underlies emergence of neuronal diversity in the neocortex.

One Sentence Summary: During corticogenesis, temporally dynamic molecular birthmarks are transmitted from progenitors to their post-mitotic progeny to generate neuronal diversity.

The cerebral cortex is a cellularly heterogeneous structure, whose neuronal circuits underlie highorder cognitive and sensorimotor information processing. During embryogenesis, distinct subtypes of glutamatergic neurons are sequentially born and differentiate from populations of progenitors located in the germinal zones below the cortex (Jabaudon 2017; Florio \& Huttner 2014). The aggregate neurogenic competence of ventricular zone progenitors (i.e. apical progenitors, APs) progresses as corticogenesis proceeds (Govindan \& Jabaudon 2017; Okamoto et al.
2016; Gao et al. 2014, Guo et al. 2013; Gaspard et al. 2007); likewise, newborn neurons transit through sequential transcriptional states as they differentiate (Zahr et al. 2018; Telley et al. 2016; Azim et al. 2009). Although the single-cell transcriptional diversity of the neocortex is increasingly well characterized (Saunders et al. 2018; Zeisel et al. 2018; Kageyama et al. 2018; Nowakowski et al. 2017; Tasic et al. 2016; Zeisel et al. 2015), little is yet known about the molecular processes driving either the progression of AP competence, or the specific 
differentiation of daughter neurons born from these progenitors at sequential embryonic ages.

To address these questions, we used FlashTag (FT), a high temporal resolution method to pulse-label APs and their daughter neurons (Telley et al. 2016; Govindan et al. 2018), to trace the transcriptional trajectories of successive generations of APs and isochronic cohorts of their daughter neurons between embryonic day (E) 12 and E15. This corresponds to the period during which APs successively generate layer (L) 6, L5, L4 and L2/3 neurons (Jabaudon 2017). Following microdissection of the putative somatosensory cortex, we collected $\mathrm{FT}^{+}$cells by FACS after either $1 \mathrm{~h}$, as APs are still dividing, $24 \mathrm{~h}$, as daughter cells are transiting through the subventricular zone, or $96 \mathrm{~h}$ (i.e. four days), once daughter neurons have entered the cortical plate (Fig. 1A and fig. S1, A and B) (Telley et al. 2016; Govindan et al. 2018). We performed single-cell RNA sequencing at each of these 3 differentiation stages and 4 embryonic ages (E12, E13, E14, and E15), which yielded a total of 2,756 quality-controlled cells across 12 conditions for analysis (fig. S1, C and D, and Methods).

Analysis of cellular transcriptional identities using t-SNE dimensionality reduction revealed two main axes of organization: a differentiation (i.e. collection time) axis and a birthdate (i.e. injection day) axis (Fig. 1B). Along the differentiation axis (Fig. 1B, left), 1 h-, 1-day- and 4-day-old cells were organized into clusters which corresponded to (1) APs, (2) basal progenitors (BPs) and 1-day-old neurons (N1d), and (3) 4-day-old neurons (N4d), as indicated by the combined expression of typespecific markers (Telley et al. 2016). Cells born at successive times of corticogenesis were organized perpendicularly to this differentiation axis, forming a birthdate axis (Fig. 1B, right). This chronotopic map was particularly apparent for APs and 1-day-old daughter cells, but less striking in 4-day-old neurons, suggesting that the salience of birthdate-related transcriptional features decreases with differentiation. Together, these data reveal two orthogonal axes of transcriptional organization: a differentiation axis, corresponding to the birth and maturation of daughter neurons, and a birthdate axis, corresponding to the temporal progression in AP transcriptional states at sequential embryonic ages. These two cardinal processes are the major source of transcriptional diversity in the developing neocortex.

We used a graph-based cluster analysis to investigate the diversity of differentiation stage- and birthdate-specific cells and identified four embryonic age-defined AP transcriptional states, as well as two embryonic age-defined basal progenitor populations, as recently reported (Yuzwa et al. 2017) (Fig. 1C). Two classes of 1-day-old neurons (N1d) could be distinguished, early-born cells (i.e. E12-E13born) and later-born cells (i.e. E14-E15-born). These two classes of neurons displayed early onset expression of deep- and superficial-layer markers, which foreshadowed their upcoming lamina-related identity (Fig. 1C). Classical deep-layer markers were also expressed by lateborn neurons (fig S2A), consistent with an initial period of mixed identity followed by molecular cross-interactions and progressive fate refinement (Zahr et al. 2018; Ozair et al. 2018; Azim et al. 2009). Accordingly, by four days of age, neurons with mutually-exclusive expression of classical lamina-specific markers such as Bcll1b (an L5 marker), Rorb (L4) and Cux1 (L2/3) emerged. Of note, GABAergic interneurons were also identified (Fig. $1 \mathrm{C}$ and fig. S2B), likely corresponding to cells migrating into the dorsal pallium after FT labeling of their progenitors in the ventral pallium (Govindan et al. 2018; Wamsley \& Fishell 2017; Marin 2013). Astrocytes, corresponding to 4-day-old daughter cells of E15 APs (Minocha et al. 2017; Cahoy et al. 2008) were also present (Fig. $1 \mathrm{C}$ and fig. S2C). These two cell-types were not further investigated in this study. Differential expression analysis identified type-enriched transcripts (Fig. 1D) whose temporal patterns of expression were confirmed using in situ hybridization (Fig. 1E; figs. S3 and S4; ISH; Allen Developing Mouse Brain Atlas). Together, these results indicate that APs transit through temporally dynamic transcriptional states during corticogenesis as daughter neurons 

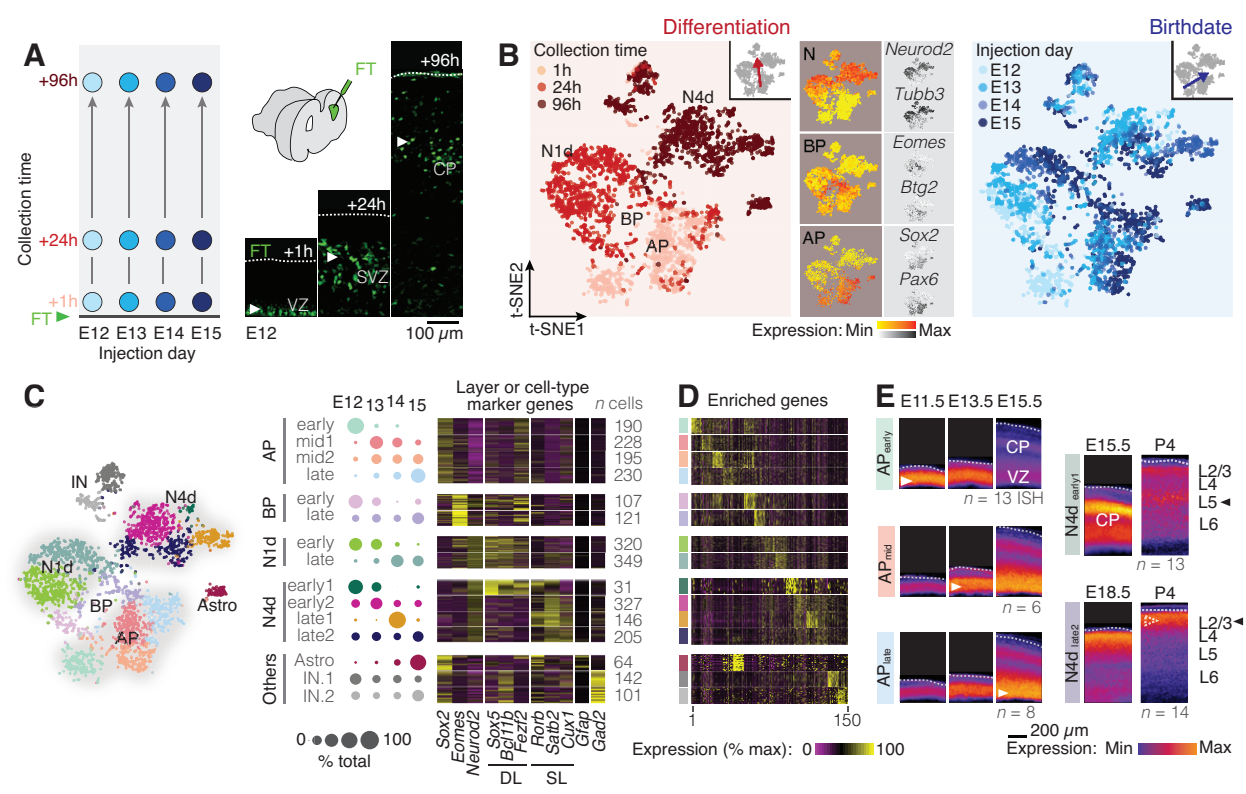

Fig. 1. Birthdate and differentiation stage-related cellular diversity in the developing neocortex. (A) Schematic illustration of the experimental procedure. M-phase APs were labeled by FT injection performed at either E12, E13, E14 or E15 and isochronic cohorts of APs and daughter cells were collected either 1, 24 or 96 hours later. (B) t-SNE representation of the single cell RNA sequencing dataset revealing the transcriptional organization of the cells according to the time at which they were collected (i.e. their differentiation status) and the day on which the injection was performed (i.e. their birthdate). APs, BPs and Ns can be distinguished by their combinatorial expression of key marker genes $(\mathrm{n}=20$ transcripts). (C) Cluster analysis reveals transcriptomically distinct and temporally dynamic cellular clusters. Cluster nomenclature reflects prevalence of the cluster at a given embryonic age (early: E12/E13, late: E14/E15). Cells in these clusters express classical layer and cell-type marker genes in accordance with their birthdate and differentiation status. (D) Expression of the top 150 most highly variable genes highlights cluster diversity. (E) Spatio-temporal expression of cluster-specific transcripts with in situ hybridization. (ISH), from the Allen Developing Mouse Brain Atlas. Color-coded images represent the average expression for representative transcripts (see also Supplementary Figs 3 and 4). Abbreviations: AP: apical progenitors, BP: basal progenitors, Astro: astrocyte, IN: interneurons, N1d: 1-day-old neurons, N4d: 4-day-old neurons, VZ: ventricular zone, SVZ: subventricular zone, CP: cortical plate, DL: deep layer, SL: superficial layer. 
progressively acquire more mature transcriptional features.

We used two axes of investigation to address the transcriptional dynamics of APs and differentiating neurons: on the one hand, we studied the progression in AP transcriptional states between E12 and E15 (Fig. 2), and on the other hand we studied the transcriptional differentiation of neurons born on each of these embryonic days, as shown in Fig. 3. To address the temporal progression in AP transcriptional states we used a pseudotime (i.e. pseudobirthdate) alignment approach (Mayer et al. 2018), which highlighted the chronotopic organization of these cells and identified clusters of genes with similar embryonic agedefined expression dynamics (Fig. 2, A and B; fig. S5, A and B). Several of these dynamicallyexpressed genes have previously characterized functions in the temporal regulation of progenitor competence, including the earlypeaking transcripts Hmga2 (E12 peak) and Aspm (E13 peak) and the late-peaking transcript Zbtb20 (E15) (Kishi et al. 2012; Johnson et al. 2018; Tonchev et al. 2016). Ontological analysis revealed the progression of AP functional properties during corticogenesis (Fig. 2C, fig. S5C). At early embryonic ages (E12, E13), APs were involved in largely cellautonomous tasks, such as regulation of gene expression and of chromatin structure; celldeath related processes were also prominent, suggesting some level of regulation of the size of the progenitor pool (Cunningham et al. 2013). Later in corticogenesis (E14, E15), external signaling and cell-cell interaction processes increased, as did lipid metabolism, which has been linked with progenitor fate in adult neuronal stem cells (Knobloch et al. 2013). Ion transport-related processes became more salient, in line with the role of bioelectrical parameters in the progression of AP competence (Vitali et al. 2018) and other typically neuronrelated processes involving synapses and neurotransmission increased. As further discussed below, this suggests that APs progressively acquire molecular signatures of their progeny upon repetitive rounds of cell division. Finally, glia-related processes emerged, consistent with the generation of this cell type in late corticogenesis (Jabaudon 2017; Gao et al. 2014; Guo et al. 2013). Together, these findings identify the sequential unfolding of successive transcriptional and functional programs within APs as corticogenesis proceeds.

We next examined the transcriptional programs expressed by differentiating neurons born on each embryonic age (Fig. 3). Pseudotime (i.e. pseudo-differentiation) alignment highlighted the sequential differentiation states of these cells and identified dynamically-expressed genes (Fig. 3 A-C; fig. S6, and see Methods). Clustering of these transcripts according to their expression dynamics outlined successive transcriptional waves driving differentiation (Telley et al. 2016) (Fig. 3C). The sequential unfolding of gene expression was essentially conserved across embryonic ages, as revealed by largely overlapping gene expression dynamics (Fig. 3D and fig. S6). Conserved gene expression did not simply reflect the constant activity of a small number of "pan-neuronal" genes (e.g. NeuroD2, Tubb2) but instead reflected genuinely conserved differentiation programs since over half of the expressed genes had highly conserved expression dynamics $(\mathrm{R}>0.8$, Fig. 3D). Accordingly, gene ontologies within waves were conserved across embryonic ages (Fig. $3 \mathrm{E})$. Thus, in contrast to the programs driving the temporal progression in AP identity, the differentiation programs of daughter neurons are largely conserved across embryonic ages, despite the distinct identities these daughter neurons acquire.

How then does neuronal diversity emerge? As reported above, the chronotopic arrangement of APs is also present in their 1-day old progeny (Fig. 1B). This suggests that embryonic age-dependent AP transcriptional programs are transmitted to their progeny to generate successive initial neuronal identities. To investigate this possibility, we next determined how dynamic transcriptional networks emerge in single cells during corticogenesis. We used a machine learning strategy to classify cells based on (1) their birthdate and (2) their differentiation status, 

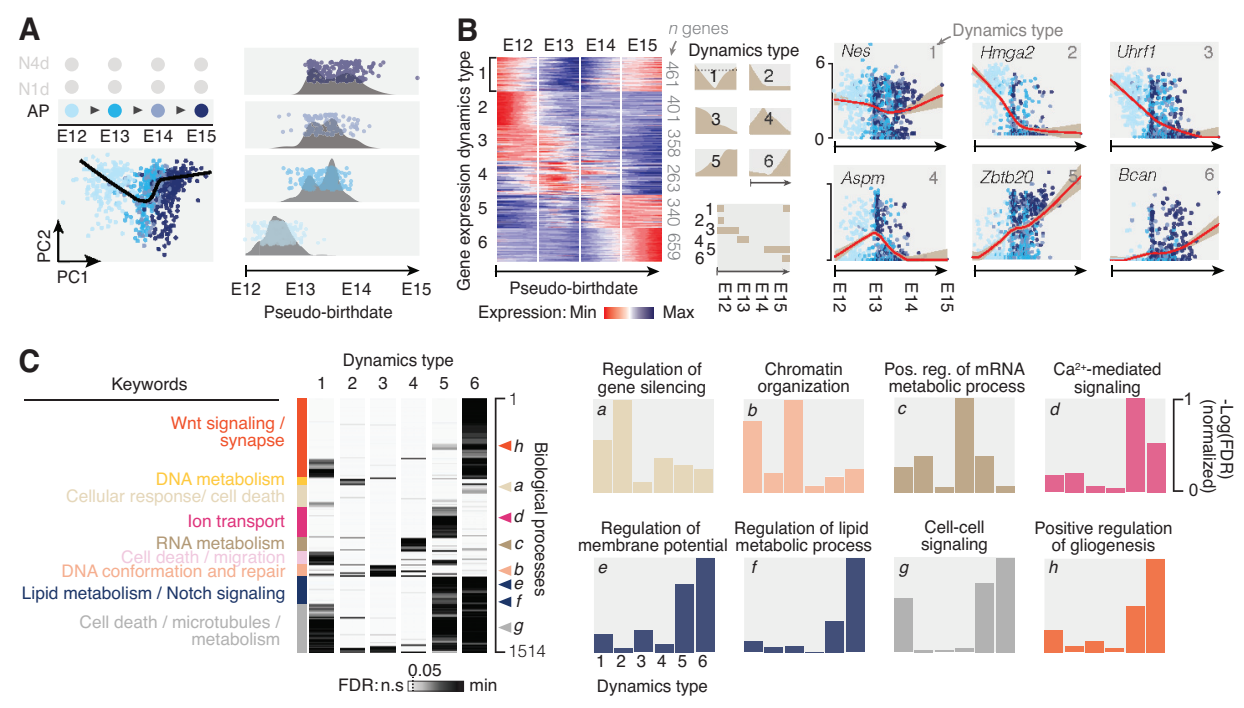

Fig. 2. Apical progenitors transit through sequential transcriptional states during corticogenesis. (A) Principal component analysis (PCA) of AP transcriptional identity showing chronotopic organization along a birthdate axis (i.e. from E12 to E15). Cells were aligned on a pseudo-birthdate axis to trace this maturation route (black line). (B) Cluster analysis reveals distinct dynamics of AP gene expression during corticogenesis. Examples of genes for each type of dynamics are provided on the right. (C) Examples of gene ontology processes associated with each of the expression dynamics. Descriptions of functions in the left panel summarize relevant ontologies. Abbreviations: AP: apical progenitor, N1d: 1-day-old neuron, N4d: 4-day-old neuron, VZ: ventricular zone, CP: cortical plate. 

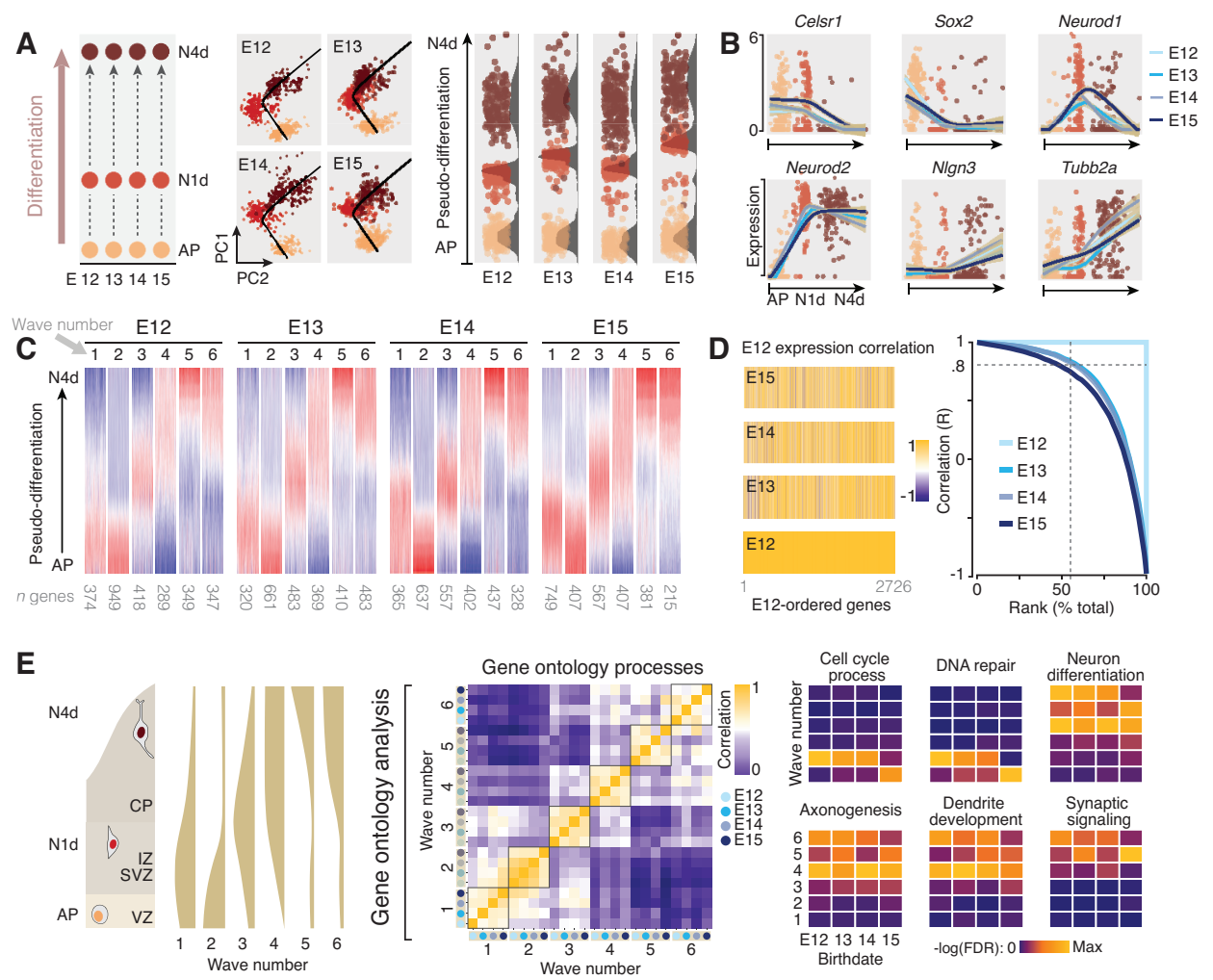

Fig. 3. Neuronal differentiation programs are conserved across corticogenesis. (A) Principal component analysis (PCA) shows that at each developmental age, cells are spontaneously organized along a differentiation axis (i.e. from AP to N1d to N4d). Cells were aligned along a pseudo-differentiation axis to trace this maturation route (black line). (B) Gene expression kinetics along the pseudo-differentiation axis at each developmental age. Displayed cells correspond to E12. (C) Clustering of the gene expression kinetics reveals sequential expression of transcriptional waves. (D) Correlation of gene kinetics considering E12 as a reference shows that most gene expression dynamics are independent of the developmental age. (E) Parallel progression of gene ontology processes associated with each transcriptional wave. Abbreviations: AP: apical progenitor, CP: cortical plate, IZ: intermediate zone, N1d: 1-day-old neuron, N4d: 4-day-old neuron, SVZ: subventricular zone, VZ: ventricular zone. 
which identified two core sets of genes ( $n=100$ per model) sufficient to classify all cells according to these two cardinal features (Fig. 4A and fig. S7A-C), many of which have been previously involved in regulating progenitor and neuronal fate (Tables 1 and 2). Birthdateassociated core genes were sequentially expressed by APs and their 1- and 4-day old progeny, directly demonstrating transmission of age-specific genesets to daughter cells (Fig. 4B, top and fig. S7D). In contrast, consistent with a consensus post-mitotic differentiation program, the dynamics of the differentiation geneset were conserved across embryonic ages (Fig. 4B, bottom). Consistent with the increase in neuronrelated ontologies in APs noted above, expression of the neuronal differentiation geneset progressively increased in APs as corticogenesis unfolded (fig. S7E and F); the latter cells thus become progressively "neuralized" as they give rise to successive generations of post-mitotic daughter cells. Taken together, these data reveal that neurontype-specific identities emerge from temporally-defined, AP-derived transcriptional ground states onto which essentially conserved post-mitotic differentiation programs are applied (Fig. 4C).

We combined the two aforementioned models to identify birthdate- and differentiation stage-related patterns of gene expression. Based on the combined expression of the core genes of the two models, each cell was assigned a birthdate score and differentiation score. Cells were then embedded within a two-dimensional matrix, allowing the display of gene expression profiles as chrono-typic transcriptional maps (Fig. 5A) (Nowakowski et al. 2017). This approach revealed a variety of dynamicallyregulated transcriptional patterns, including within single families of genes (Fig. 5B and fig. S8). To identify archetypical features of gene expression, we performed a t-SNE-based cluster analysis of all transcriptional maps, revealing canonical clusters of genes with similar expression dynamics (Fig. 5C). Genes within each of these canonical clusters shared common functions, and the distinct clusters were functionally specialized (Fig. 5D and fig. S9). This suggests that these transcriptional clusters represent functional units orchestrating the unfolding of cellular processes during corticogenesis. To substantiate this possibility, we selected one early and one late AP transcriptional process and assessed its functional outcome (Fig. 5E and F). Expression of the Polycomb Repressive Complex 2 (PRC2), which regulates histone methylation in neural progenitors (Pereira et al., 2010), provided a first example: all three subunits of the complex were co-expressed in APs early in corticogenesis, and the $\mathrm{H} 3 \mathrm{~K} 27 \mathrm{me} 3$ signature mark of PRC2 had corresponding dynamics on target sites, demonstrating temporally-gated functional activity (Fig. 5E). Expression of the glutamate transporter transcript Slcla3 (Glast) constituted a second example: Glast increased in APs as glutamatergic neurotransmission developed in the cortical plate. Pharmacological blockade of this transporter increased glutamate levels at late, but not early embryonic stages, consistent with a dynamic bioelectrical control over AP properties during corticogenesis (Fig. 5F) (Vitali et al. 2018).

Together, our findings identify a combinatorial process in which type-specific neuronal identity emerges from the apposition of generic differentiation programs onto embryonic age-dependent, AP-derived transcriptional states. In this scenario, neuronal differentiation essentially corresponds to the implementation of programs coding for generic neuronal features (e.g. neurites, neurotransmission) onto temporally-defined initial transcriptional states. This process is reminiscent of how neuron diversity is generated in evolutionary older brain regions such as the subpallium or spinal cord ( Mayer et al. 2018; Mi et al. 2018; Nowakowski et al. 2017; Dasen \& Jessell 2009), with the difference that in these regions, distinctions in initial neuronal states reflect a predominantly spatial rather than temporal distribution of molecularly distinct progenitors. There thus appears to be at least two ways to generate cellular diversity: spatial patterning of molecularly distinct progenitors (e.g. subpallium, spinal cord), and temporal patterning, as revealed here. In evolutionary terms, temporal patterning may have been 

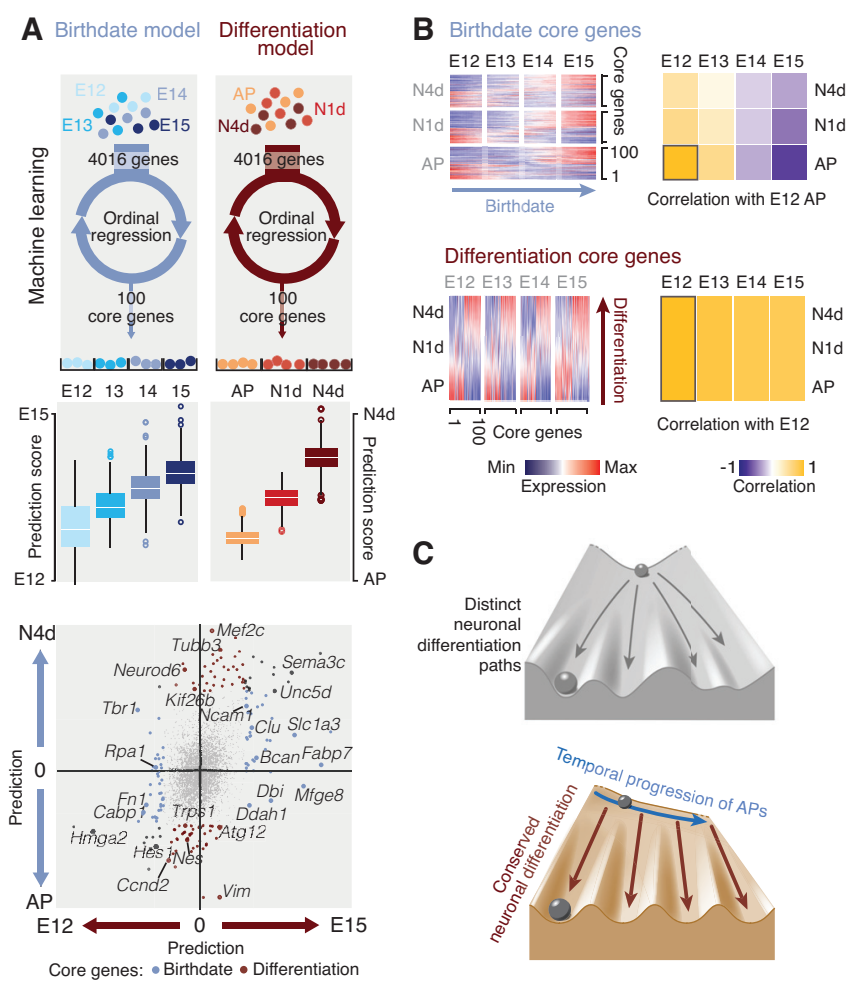

Fig. 4. Temporally progressing AP transcriptional states interact with conserved differentiation programs to generate neuronal diversity. (A) Machine learning approach used to identify a core set of genes which can classify cells based on their date of birth (top left) and differentiation status (top right). Center: Model performance using actual dataset. Box plot: median \pm SEM. Bottom: Weight of the core genes in predicting birthdate and differentiation status. See also tables S1 and S2. (B) Top: Birthdate-associated core genes are temporally dynamic and daughter cells acquire embryonic stage-specific transcriptional birthmarks. See also Supplementary Fig.7D. Bottom: In contrast, differentiation status-associated core genes are conserved across corticogenesis. (C) Schematic representation of the findings: in the classical Waddington epigenetic model (top) cellular diversity emerges through distinct developmental trajectories. The current data shows that instead, in the neocortex, developmental trajectories are conserved, but that initial ground states are temporally dynamic. Abbreviations: AP: apical progenitor, N1d: 1-day-old neuron, N4d: 4-day-old neuron. 

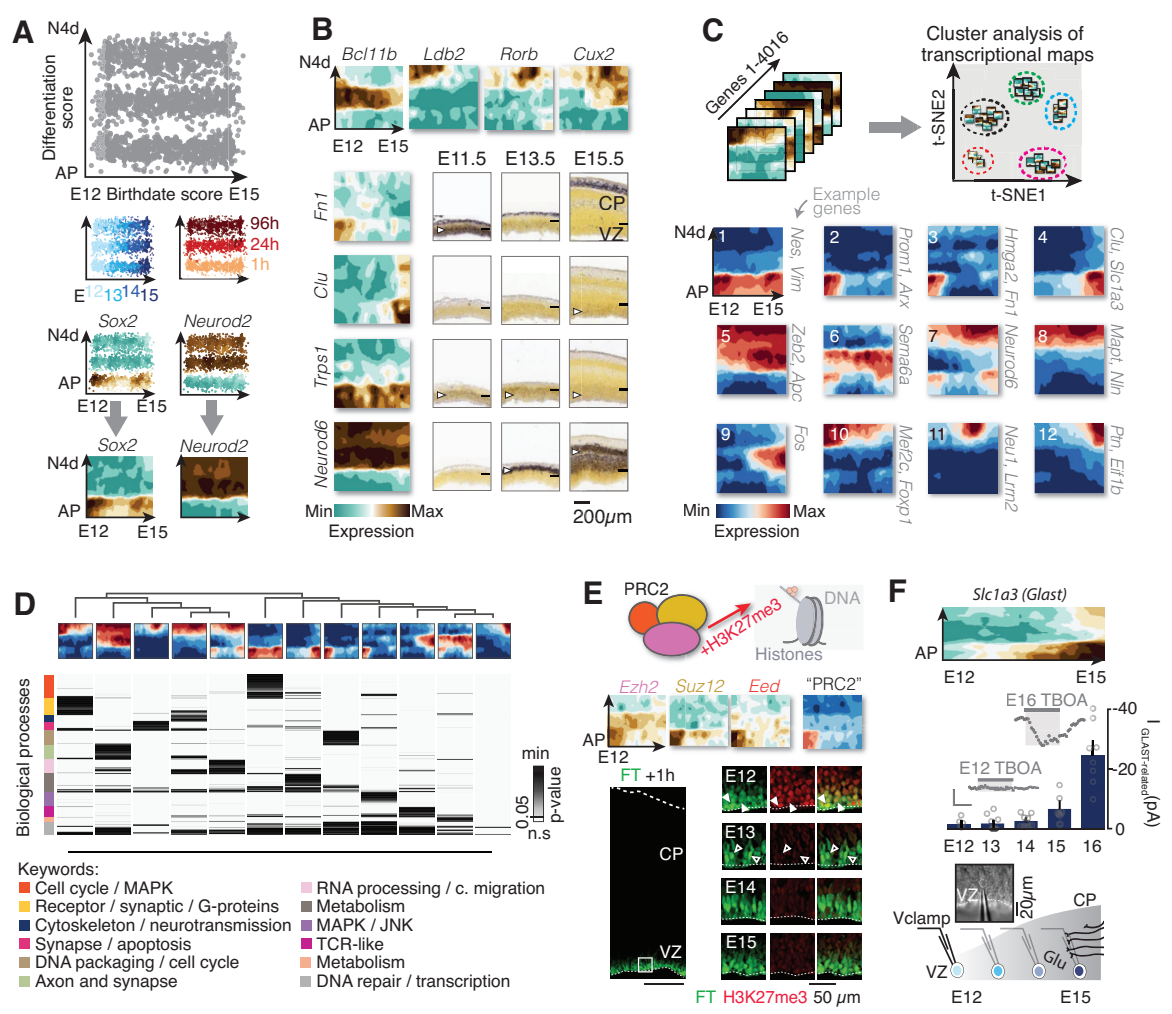

Fig. 5. Dynamic transcriptional mapping of corticogenesis. (A) Cellular map in which cells are displayed based on their combined expression of the birthdate and differentiation status core genes presented in Fig. 4. Dynamic expression of genes ("transcriptional maps") throughout corticogenesis can be determined based on this template (as exemplified with Sox2 and NeuroD2). (B) Example of transcriptional landscapes for select genes, and corresponding ISH validation from the Allen Developing Mouse Brain Atlas (www.brain-map.org). (C) Canonical transcriptional maps can be identified by t-SNE clustering of the maps of individual genes. (D) Genes belonging to each of the clusters have converging and specialized ontologies. (E) The distinct components of the polycomb repressive complex 2 have equivalent chrono-typic expression profiles; functional interrogation of this complex with the H3K27me3 mark reveals a corresponding methylation enrichment in E12 APs. (F) The glutamate transporter transcript Slc1a3 (Glast) is expressed by APs late in corticogenesis. Blockade of GLAST with TBOA (Jabaudon et al. 1999) increases extracellular glutamate at late but not early embryonic ages, as detected by activation of ionotropic glutamate receptors in patch-clamped APs. Scale bars: 5 min; 20 pA. Abbreviations: AP: apical progenitor, N4d: 4-day-old neuron, PRC2: Polycomb Repressive Complex 2, CP: cortical plate, VZ: ventricular zone. 
selected as the primary mode of neuron production in the neocortex because it allows the generation of a large spectrum of cell types at low spatial cost. How temporal birthmarks are transmitted from APs to daughter neurons is unclear, but the strong temporal control over epigenetic processes identified here suggests that transmission of 3D chromatin features may be involved. In addition, the passive transmission of cytoplasmic RNA into daughter neurons along with post-transcriptional events could also contribute (Zahr et al. 2018; Yoon et al. 2017, 2018). As previously reported, we find that newborn neurons initially express a combination of classical lamina type-specific markers, a process which has been termed "transcriptional priming" and is also found in other organs such as the hematopoietic system ( Zahr et al. 2018; Azim et al. 2009; Hu et al. 1997). Our findings thus do not exclude the contribution of post-mitotic processes to fate refinement (Zahr et al. 2018; Ozair et al. 2018; Mayer et al. 2018; Telley \& Jabaudon 2018), but AP-derived, temporally-regulated processes appear to have a primordial role in defining initial neuronal identity. Although still present in 4-day-old neurons, temporal birthmarks fade with differentiation. At these later stages, activity-dependent programs may be progressively implemented in interaction with the environment to complement and eventually override earlier transcriptional processes, culminating in the generation of the full complement of cells required for functional cortical circuits.

\section{Acknowledgments}

We thank A. Benoit and the Genomics Platform and FACS Facility of the University of Geneva for technical assistance; and H. Wu, E. Azim, O. Raineteau, D. Silver and the members of the Jabaudon laboratory for comments on the manuscript. Illustration in panel $4 \mathrm{C}$ : www.lagraphisterie.fr. Work in the Jabaudon laboratory is supported by the Swiss National Science Foundation and the Carigest Foundation. DJ and AD are supported by the Fondation privée des HUG. G.A. is a $\mathrm{PhD}$ student from the F.R.S-F.N.R.S and is supported by the Fonds Léon Fredericq. The LN laboratory is funded by the F.R.S.-FNRS (EOS O019118F-RG36; CDR J.0028.18; PDR T.0073.15) the Fonds Léon Fredericq, the Fondation Médicale Reine Elisabeth, and the Fondation Simone et Pierre Clerdent. A.D. is funded by the Swiss National Science Foundation and the National Center of Competence in Research (NCCR) Synapsy.

\section{Author contributions}

LT and GA performed the experiments with the help of PO and IV. SF performed the electrophysiology experiments. LT, GA and JP performed the bioinformatic analysis. GA, DJ and LT wrote the manuscript with the help of $\mathrm{AD}$ and $\mathrm{LN}$.

\section{Competing interests}

None.

\section{Data and materials availability}

Annotated data will be available upon manuscript publication. The supplementary materials contain additional data.

\section{References}

Azim, E. et al., 2009. Lmo4 and Clim1 progressively delineate cortical projection neuron subtypes during development. Cerebral Cortex, 19 Suppl 1(suppl_1), pp.i62-9.

Cahoy, J.D. et al., 2008. A transcriptome database for astrocytes, neurons, and oligodendrocytes: a new resource for understanding brain development and function. Journal of Neuroscience, 28(1), pp.264-278.

Cunningham, C.L., Martinez-Cerdeño, V. \& Noctor, S.C., 2013. Microglia regulate the number of neural precursor cells in the developing cerebral cortex. Journal of Neuroscience, 33(10), pp.42164233.

Dasen, J.S. \& Jessell, T.M., 2009. Hox networks and the origins of motor neuron diversity. Development of Neural Circuitry, 88, pp.169-200.

Florio, M. \& Huttner, W.B., 2014. Neural progenitors, neurogenesis and the evolution of the neocortex. Development, 141(11), pp.2182-2194. 
Gao, P. et al., 2014. Deterministic progenitor behavior and unitary production of neurons in the neocortex. Cell, 159, 775-788.

Gaspard, N. et al., 2008. An intrinsic mechanism of corticogenesis from embryonic stem cells. Nature, 455, 351-357.

Govindan, S. \& Jabaudon, D., 2017. Coupling progenitor and neuronal diversity in the developing neocortex. FEBS Lett. 591(24), pp. 3960-3977.

Govindan, S. et al., 2018. In vivo pulse labeling of isochronic cohorts of cells in the central nervous system using FlashTag. Nature Protocols. DOI : 10.1038/s41596-018-0038-1.

Guo, C. et al., 2013. Fezf2 expression identifies a multipotent progenitor for neocortical projection neurons, astrocytes, and oligodendrocytes. Neuron, 80, 1167-1174.

$\mathrm{Hu}, \mathrm{M}$. et al., 1997. Multilineage gene expression precedes commitment in the hemopoietic system. Genes \& Development, 11(6), pp.774-785.

Jabaudon, D., 2017. Fate and freedom in developing neocortical circuits. Nature Communications, 8, p.16042.

Jabaudon, D. et al., 1999. Inhibition of uptake unmasks rapid extracellular turnover of glutamate of nonvesicular origin. Proceedings of the National Academy of Sciences of the United States of America, 96(15), pp.8733-8738.

Johnson, M.B. et al., 2018. Aspm knockout ferret reveals an evolutionary mechanism governing cerebral cortical size. Nature, 556(7701), pp.370375.

Kageyama, J. et al., 2018. ShinyCortex: Exploring single-cell transcriptome data from the developing human cortex. Frontiers in Neuroscience, 12, p.315.

Kishi, Y. et al., 2012. HMGA regulates the global chromatin state and neurogenic potential in neocortical precursor cells. Nature Neuroscience, 15(8), pp.1127-1133.

Knobloch, M. et al., 2013. Metabolic control of adult neural stem cell activity by Fasn-dependent lipogenesis. Nature, 493(7431), pp.226-230.

Marin, O., 2013. Cellular and molecular mechanisms controlling the migration of neocortical interneurons. European Journal of Neuroscience, 38(1), pp.2019-2029.

Mayer, C. et al., 2018. Developmental diversification of cortical inhibitory interneurons. Nature, 555(7697), pp.457-462.
Mi, D. et al., 2018. Early emergence of cortical interneuron diversity in the mouse embryo. Science (New York, NY), 360(6384), pp.81-85.

Minocha, S. et al., 2017. Nkx2.1 regulates the generation of telencephalic astrocytes during embryonic development. Scientific Reports, 7(1), p.43093.

Nowakowski, T.J. et al., 2017. Spatiotemporal gene expression trajectories reveal developmental hierarchies of the human cortex. Science, 358(6368), pp.1318-1323.

Okamoto, M. et al., (2016). Cell-cycle-independent transitions in temporal identity of mammalian neural progenitor cells. Nature Communications, 7, 11349 .

Ozair, M.Z. et al., 2018. hPSC modeling reveals that fate selection of cortical deep projection neurons occurs in the subplate. Cell Stem Cell, 23(1), pp.60-73.e6.

Pereira, J.D. et al., 2010. Ezh2, the histone methyltransferase of PRC2, regulates the balance between self-renewal and differentiation in the cerebral cortex. Proceedings of the National Academy of Sciences of the United States of America, 107(36), pp.15957-15962.

Saunders, A. et al., 2018. Molecular diversity and specializations among the cells of the adult mouse brain. Cell, 174(4), pp.1015-1030.e16.

Tasic, B. et al., 2016. Adult mouse cortical cell taxonomy revealed by single cell transcriptomics. Nature Neuroscience, 19(2), pp.335-346.

Telley, L. \& Jabaudon, D., 2018. A mixed model of neuronal diversity. Nature, 555(7697), pp.452454.

Telley, L. et al., 2016. Sequential transcriptional waves direct the differentiation of newborn neurons in the mouse neocortex. Science, 351(6280), pp.1443-1446.

Tonchev, A.B. et al., 2016. Zbtb20 modulates the sequential generation of neuronal layers in developing cortex. Molecular Brain, 9(1), p.65.

Vitali, I. et al., 2018. Progenitor hyperpolarization regulates the sequential generation of neuronal subtypes in the developing neocortex. Cell, 174(5), pp.1264-1276.e15.

Wamsley, B. \& Fishell, G., 2017. Genetic and activity-dependent mechanisms underlying interneuron diversity. Nature Reviews Neuroscience, 18(5), pp.299-309. 
Yoon, K.-J. et al., 2017. Temporal control of mammalian cortical neurogenesis by m6a methylation. Cell, 171(4), pp.877-889.e17.

Yoon, K.-J. et al., 2018. Epigenetics and epitranscriptomics in temporal patterning of cortical neural progenitor competence. Journal of Cell Biology, 217(6), pp.1901-1914.

Yuzwa, S.A. et al., 2017. Developmental emergence of adult neural stem cells as revealed by single-cell transcriptional profiling. Cell Reports, 21(13), pp.3970-3986.

Zahr, S.K. et al., 2018. A translational repression complex in developing mammalian neural stem cells that regulates neuronal specification. Neuron, 97(3), pp.520-537.e6.

Zeisel, A. et al., 2015. Brain structure. Cell types in the mouse cortex and hippocampus revealed by single-cell RNA-seq. Science, 347(6226), pp.1138-1142.

Zeisel, A. et al., 2018. Molecular architecture of the mouse nervous system. Cell, 174(4), pp.9991014.e22. 
bioRxiv preprint doi: https://doi.org/10.1101/409458; this version posted September 6, 2018. The copyright holder for this preprint (which was not certified by peer review) is the author/funder. All rights reserved. No reuse allowed without permission.

Table 1: Selection of characterized genes from the birthdate model

\begin{tabular}{|c|c|c|c|c|}
\hline $\begin{array}{l}\text { Gene } \\
\text { symbol }\end{array}$ & $\begin{array}{l}\text { Enriched } \\
\text { in }\end{array}$ & $\begin{array}{l}\text { Gene } \\
\text { weight }\end{array}$ & Function & References (PMID) \\
\hline Hmga2 & Early & -6.25 & $\begin{array}{l}\text { DNA-binding, chromatin-related. KO in early-stage cortical progenitors reduces neurogenic } \\
\text { potential }\end{array}$ & $22797695 / 18957199$ \\
\hline Tbr1 & Early & -3.65 & TF. Instructs the laminar location and identity of deep layer neurons & 16858776 / 20615956 \\
\hline Top2a & Early & -3.36 & DNA topoisomerase. KO leads to laminar dysruptions in the cerebral cortex & 12773624 \\
\hline$B c 111 b$ & Early & -3.13 & CTIP2, a TF. Marker of L5B neurons & $15664173 / 18678899$ \\
\hline Hzafz & Early & -2.91 & Histone protein. KO results in enhanced proliferation of progenitors and reduced differentiation. & 29294103 \\
\hline Gadd45g & Early & -2.78 & DNA demethylation. Direct target of Pax6. & 19521500 \\
\hline Hes1 & Early & -2.58 & Transcriptional repressor. Represses precocious neuronal commitment of cortical progenitors & 10627606 \\
\hline Filip1 & Early & -2.24 & Filamin-interacting protein. Controls the radial migration of newborn cortical neurons & 12055638 \\
\hline Sox 5 & Early & -2.15 & TF. Control the timing of sequential generation of corticofugal neurons subtypes & $18215621 / 18840685$ \\
\hline Ptprz1 & Late & 2.73 & Receptor tyrosine phosphatase. In human oRGs; development of OPCs. & $26406371 / 21969550$ \\
\hline Cttnbp2 & Late & 2.85 & Dendritogenesis & 23015759 \\
\hline Sparcl1 & Late & 2.88 & Surface protein. Terminal migration of neurons. Astrocyte marker & 14715135 \\
\hline Glra2 & Late & 2.91 & Glycin receptor subunit. Regulates BP generation & 24926615 \\
\hline Trim9 & Late & 3.02 & Ubiquitin ligase. Netrin1 signaling-associated; regulates neuronal morphogenesis & 28701345 \\
\hline Nr2f1 & Late & 3.09 & TF, COUP-TFI. Neuronal differentiation & 25476200 \\
\hline Trim2 & Late & 3.36 & Regulates axonal mrophogenesis & 20796172 \\
\hline Tnc & Late & 3.86 & Extracellular matrix protein. oRG marker & 26406371 \\
\hline Chl1 & Late & 3.92 & $\begin{array}{l}\text { ECM and cell adhesion protein. Inhibits Erk1/2- MAPK signaling and reduces progenitor } \\
\text { proliferation }\end{array}$ & $\begin{array}{l}15504324 / 20933598 / \\
18077678\end{array}$ \\
\hline Unc5d & Late & 4.36 & Netrin receptor. Expressed in $L 4$ neurons; mediates neuronal survival & $21216843 / 18469807$ \\
\hline Sema3c & Late & 5.36 & Semaphorin. Required for migration of superficial layer neurons & 26182416 \\
\hline Zbtb20 & Late & 7.04 & Regulator for the generation of layer-specific neuronal identities; neuronal maturation & 27282384 / 24828045 \\
\hline
\end{tabular}


bioRxiv preprint doi: https://doi.org/10.1101/409458; this version posted September 6, 2018. The copyright holder for this preprint (which was not certified by peer review) is the author/funder. All rights reserved. No reuse allowed without permission.

Table 2: Selection of characterized genes from the differentiation model

\begin{tabular}{|c|c|c|c|c|}
\hline $\begin{array}{l}\text { Gene } \\
\text { symbol }\end{array}$ & $\begin{array}{l}\text { Enriched } \\
\text { in }\end{array}$ & $\begin{array}{l}\text { Gene } \\
\text { weight }\end{array}$ & Function & References (PMID) \\
\hline Vim & AP & -4.85 & Intermediate filament protein. Classical marker of radial glia cells & Multiple references \\
\hline Ccnd2 & AP & -3.43 & Cylin D2. Required for generation of BPs from APs & 19641124 \\
\hline Chd7 & AP & -3.17 & Chromatin remodeler. Regulates AP proliferation; interacts with Sox2 & $27955690 / 21532573$ \\
\hline Boc & AP & -3.10 & SHH co-receptor. Regulates neuronal differentiation from cortical progenitor cells & 27871935 \\
\hline Cdon & AP & -3.07 & SHH co-receptor. Regulates cortical progenitor proliferation and neuronal differentiation & 16648472 \\
\hline H2afz & AP & -2.98 & Histone protein. $\mathrm{KO}$ results in enhanced proliferation of progenitors and reduced differentiation. & 29294103 \\
\hline Hes1 & AP & -2.88 & Transcriptional repressor. Represses precocious neuronal commitment of cortical progenitors & 10627606 \\
\hline Nes & AP & -2.65 & Intermediate filament protein. Classical marker of radial glia cells & Multiple references \\
\hline Rapgef6 & AP & -2.59 & GTPase. Reported to maintain the apical surface adherens junction in cortical progenitors. & $27390776 / 28917843$ \\
\hline$B c 171 b$ & AP & -2.55 & CTIP2, a TF. Marker of L5B neurons & $15664173 / 18678899$ \\
\hline Nfia & AP & -2.51 & TF. Represses Notch pathway to initiate neuronal differentiation & 20610746 \\
\hline Qk & AP & -2.30 & Involved in neuron-glia fate decisions & 9778149 \\
\hline $\operatorname{Arx}$ & AP & -2.11 & TF. Regulates AP proliferation and generation of BPs & $23968833 / 18509041$ \\
\hline Unc5d & $\mathrm{N}$ & 3.06 & Netrin receptor. Expressed in $L 4$ neurons; mediates neuronal survival & $21216843 / 18469807$ \\
\hline Aff2 & $\mathrm{N}$ & 3.08 & TF, transiently expressed in SVZ. Reported role in lymphcyte differentiation & 12079280 \\
\hline Sparcl1 & $\mathrm{N}$ & 3.10 & SVZ protein; obligatory binding partner of the neurite outgrowth-promoting factor pleiotrophin & 28823557 \\
\hline Syt4 & $\mathrm{N}$ & 3.23 & Syntaxin 4 , retrograde synaptic signalling & 23522040 \\
\hline Celf2 & $\mathrm{N}$ & 3.25 & Regulation of RNA splicing & 11158314 \\
\hline Nlgn 3 & $\mathrm{~N}$ & 3.28 & Neuroligin 3, synaptic adhesion molecule & 26235839 \\
\hline Sox11 & $\mathrm{N}$ & 3.30 & $\mathrm{TF}$, interacts with LHX2 & 28053041 \\
\hline Trim67 & $\mathrm{N}$ & 3.36 & KO has CNS defects including decreased size of callosum & 26235839 \\
\hline Nrxn1 & $\mathrm{N}$ & 3.37 & Neurexin 1 , synaptic protein & 28013231 \\
\hline Zbtb18 & $\mathrm{N}$ & 3.40 & Disruption of superficial cortical layers in KO & 28053041 \\
\hline Dpysl3 & $\mathrm{N}$ & 3.56 & Axonal guidance and outgrowth & 10504203 \\
\hline Bcl11a & $\mathrm{N}$ & 3.63 & TF. Controls the migration of cortical neurons with Sema3c; settles identity of corticofugal neurons & $26182416 / 25972180$ \\
\hline Sema3c & $\mathrm{N}$ & 3.76 & Semaphorin. Required for migration of superficial layer neurons & 26182416 \\
\hline Neurod6 & $\mathrm{N}$ & 3.88 & TF. Classical neuronal marker & Multiple references \\
\hline Satb2 & $\mathrm{N}$ & 3.88 & $\begin{array}{l}\text { TF. Regulates differentiation of callosal projection neurons; mutually repressive interactions with } \\
\text { Fezf2 }\end{array}$ & $\begin{array}{l}26324926 / 25037921 / 18255031 / \\
18255030\end{array}$ \\
\hline$D c x$ & $\mathrm{~N}$ & 3.98 & Microtubule associated protein. Critical for neuronal migration and proper cortical layering & $\begin{array}{l}10399932 \text { / } 10399933 / 14625554 / \\
12764037\end{array}$ \\
\hline Clstn2 & $\mathrm{N}$ & 4.11 & Excitatory synapse transmission & 12498782 / 28912692 \\
\hline Tuba1a & $\mathrm{N}$ & 4.11 & Tubulin-related. Mutation causes lisencephaly & 20466733 \\
\hline Arpp21 & $\mathrm{N}$ & 4.23 & RNA-binding; controls neuronal excitability and dendritic morphology in neocortex & 29581509 \\
\hline Gpm6a & $\mathrm{N}$ & 4.34 & Neuronal differentiation and migration of neuronal stem cells & 19298174 \\
\hline Smarca2 & $\mathrm{N}$ & 4.37 & Chromatin-remodeling; activation of Neurod2 and Ngn & $11134956 / 15576411$ \\
\hline Rtn1 & $\mathrm{N}$ & 4.38 & ER-related; involved in neuronal differentiation; used as a marker & 9560466 \\
\hline Neurod2 & $\mathrm{N}$ & 4.46 & Neuronal specific genes, induces premature exit from cell cycle & 26940868 \\
\hline Zbtb20 & $\mathrm{N}$ & 4.51 & Regulator for the generation of layer-specific neuronal identities; neuronal maturation & 27282384 / 24828045 \\
\hline Gria2 & $\mathrm{N}$ & 4.57 & AMPA receptor subunit & Multiple references \\
\hline Tubb3 & $\mathrm{N}$ & 4.63 & Tubulin-related. Mutation causes abnormal cortical development & 30016746 \\
\hline Mef2c & $\mathrm{N}$ & 5.37 & Activity-dependent TF. Regulates synaptogenesis & 27989458 \\
\hline
\end{tabular}




\section{Materials and Methods}

$\underline{\text { Mice }}$

All experiments were approved by the Geneva Cantonal Veterinary Authorities, Switzerland. To avoid developmental variability between embryos, three hour-time-mated pregnant CD1 mice were purchased from Charles River Laboratories. Embryonic day (E) 0 was established as the time of detection of the vaginal plug. Both female and male embryos were analyzed in this study.

\section{$\underline{\text { In utero FlashTag injection }}$}

FlashTag (FT) injections were performed at E12, E13, E14 or E15, as previously described (Telley et al. 2016; Govindan et al. 2018). Briefly, pregnant females were anaesthetized with isoflurane, treated with Temgesic (Reckitt Benckiser, Switzerland) and the uterine horn was exposed following an abdominal incision. Half a microliter of $10 \mathrm{mM}$ of a carboxyfluorescein succinimidyl ester (i.e. FlashTag, CellTrace ${ }^{\mathrm{TM}}$ CFSE, Life Technologies, \#C34554) was injected into the lateral cerebral ventricle of the embryos. The abdominal wall was then closed and the embryos were let to develop until collection.

\section{Immunofluorescence and imaging}

Tissue processing: Embryonic brains were dissected in a phosphate-buffered saline (PBS) solution, fixed in $4 \%$ paraformaldehyde (PFA) overnight at $4{ }^{\circ} \mathrm{C}$ then cryoprotected in PBS-sucrose $30 \%$ overnight at $4^{\circ} \mathrm{C}$ before embedding in OCT and freezing on dry ice. On-slide coronal brain sections with a thickness of $14 \mu \mathrm{m}$ were performed using a cryostat.

Immunofluorescence on brain sections: Brain sections were post-fixed 10 min in 4\% PFA, washed three times in PBS, incubated $30 \mathrm{~min}$ at $85{ }^{\circ} \mathrm{C}$ in citrate buffer solution and washed 3 times in PBS prior to a 1 -hour incubation in blocking solution $(10 \%$ horse serum $-0,5 \%$ Triton $\mathrm{X}-100$ diluted in PBS) at room temperature. Slides were then incubated overnight at $4^{\circ} \mathrm{C}$ with primary antibodies. Next, slides were washed 3 times in PBS and incubated 2 hours at room temperature with respective secondary antibodies (1:500) before mounting with Fluoromount (Sigma). Primary antibodies used: rabbit anti-pH3 (1:500, Abcam, \#AB5176), rabbit anti-H3K27me3 (1:500, Millipore, 07-449).

Imaging: All images were acquired on LSM 700 confocal laser scanning microscope (Carl Zeiss). The putative primary somatosensory (S1) cortex was used as a region of study. The ImageJ software was further used for downstream image processing.

\section{$\underline{\text { In situ hybridization image processing }}$}

All in situ hybridizations were retrieved from the Allen Developing Mouse Brain Atlas (www.brainmap.org) and uniformly zoomed to the putative S1 neocortical region. For the illustrations Fig. 1E and figs S4, S5C, S7C the images were aligned and stacked. The mean intensity level of the Z projection was calculated on ImageJ. The resulting layout was artificially colored using the "Fire" mode of Image J.

\section{scRNAseq experiment}

Cell dissociation and FAC-sorting: Pregnant females were sacrificed either 1, 24 or 96 hours after FT injection. As previously described (Telley et al. 2016; Govindan et al. 2018), embryonic brains were 
extracted in ice-cold HBSS, embedded in 4\% agar low-melt and sectioned coronally at $300 \mu \mathrm{m}$ using a vibrating microtome (Leica, \#VT100S). The putative S1 cortical region was microdissected under a stereomicroscope and incubated in $0.05 \%$ trypsin at $37^{\circ} \mathrm{C}$ for 5 minutes. Following tissue digestion, fetal bovine serum was added to the mix and cells were manually dissociated via up-and-down pipetting. Cells were centrifuged $5 \mathrm{~min}$ at $300 \mathrm{G}$ and the pellet was suspended in $1 \mathrm{ml}$ of HBSS then passed on a $70 \mu \mathrm{m}$ cell strainer. $\mathrm{FT}^{+}$cells, gated to include only the top $5 \%$ brightest cells (Telley et al. 2016; Govindan et al. 2018), were finally FAC-sorted on a MoFloAstrios device (Beckman).

Single-cell RNA capture and sequencing: FAC-sorted $\mathrm{FT}^{+}$cells $(18 \mu \mathrm{l})$ were mixed with the C1 Suspension Reagent ( $2 \mu \mathrm{l}$; Fluidigm) yielding a total of $20 \mu \mathrm{l}$ of cell suspension mix with $\sim 500$ cells / $\mu 1$. The cell suspension mix was loaded on a C1 Single-Cell AutoPrep integrated fluidic circuit (IFC) designed for 10- to 17- $\mu \mathrm{m}$ cells (HT-800, Fluidigm \#100-57-80). cDNA synthesis and preamplification was processed following the manufacturer's instructions (C1 system, Fluidigm) and captured cells were imaged using the ImageXpress ${ }^{\circledR}$ Micro Widefield High Content Screening System (Molecular Devices ${ }^{\circledR}$ ). Single cell RNA-sequencing libraries of the cDNA were prepared using Nextera XT DNA library prep kit (Illumina). Libraries were multi-plexed and sequenced according to the manufacturer's recommendations with paired-end reads using HiSeq2500 plat-form (Illumina) with an expected depth of $1 \mathrm{M}$ reads per single cell, and a final mapping read length of $70 \mathrm{bp}$. All the single cell RNA capture and sequencing experiments were performed within the Genomics Core Facility of the University of Geneva. The sequenced reads were aligned to the mouse genome (GRCm38) using the read-mapping algorithm TopHat. Unique Molecular Identifiers (UMI) sequenced in the first reads were used to correct for cDNA PCR amplification biases. Duplicated reads were identified and corrected using the deduplication step from the UMI-tools algorithm (doi:10.1101/gr.209601.116). The number of reads per transcript was calculated with the open-source HTSeq Python library. All the analyses were computed on the Vital-It cluster administered by the Swiss Institute of Bioinformatics.

\section{$\underline{\text { scRNAseq analysis }}$}

Cell filtering: Doublet cells identified on the Fluidigm C1 plate images were excluded before initial analysis. A total of 2,906 $\mathrm{FT}^{+}$single cells were obtained $(\mathbf{F T}+\mathbf{1} \mathbf{~ h}:$ E12: 202 cells, E13: 211, E14: 135, E15: 304; FT +24 h: E12: 284 cells, E13: 286, E14: 232, E15: 217; FT + 96 h: E12: 246 cells, E13: 278, E14: 262, E15: 249). Cells expressing $<1000$ genes or $>17 \%$ of mitochondrial genes were excluded. After this step, 2'756 cells remained for analysis $(\mathbf{F T}+\mathbf{1} \mathbf{~ h}$ : E12: 189 cells, E13: 207, E14: 134, E15: 301; FT +24 h: E12: 268 cells, E13: 223, E14: 219, E15: 213; FT +96 h: E12: 244 cells, E13: 267, E14: 254, E15: 237).

Type specific transcripts: The AP, BP and N score used in Fig. 1B correspond to the mean transcript expression of the top 20 genes for AP, BP and N previously characterized in (Telley et al. 2016) were: AP: Aldoc, Pdpn, Vim, Ednrb, Ddah1, Ldha, Peg12, Wwtr1, Tspan12, Mfge8, Uhrf, Ncaph, Ndrg2, Mt1, Hk2, Psat1, Sp8, Sdc4, Dnmt3a, Notch2, Psph. BP: Btg2, Eomes, Abcg1, Kif26b, Mfap4, Corolc, Myo10, Mfng, Rprm, Chd7, Ezr, Gadd45g, Slc16a2, Heg1, Celsr1, Tead2, Cd63, Rhbdl3, Mdgal, Arrdc3. N: Myt1l, Unc5d, 1700080N15Rik, Nos1, Satb2, Ank3, Scn3a, Dscam, Cntn2, Plxna4, 9130024F11Rik, Lrrtm4, Ptprk, Nrp1, Celsr3, Rbfox1, Flrt2, Kcnq3, Kcnq2, Gm36988.

Clustering analysis was performed using the Seurat bioinformatics pipeline (https://github.com/satijalab/seurat) and is summarized here. We first created a "Seurat object" including all 2,756 cells and all genes. To remove sequencing depth biases between cells, we normalized and scaled the UMI counts using the NormalizeData (normalization.method = "LogNormalize", scale.factor $=100000$ ) combined with the ScaleData function (vars.to.regress $=$ 
c("nGene","nUMI")). We then determined the most variable genes by plotting transcripts into bins based on $\mathrm{X}$-axis (average expression) and $\mathrm{Y}$-axis (dispersion). This identified 4,016 transcripts. Parameters and cutoffs were set as follow: mean.function $=$ ExpMean, dispersion. function $=\operatorname{LogVMR}$, x.low.cutoff $=0.1$, x.high.cutoff $=8$, y.cutoff $=0.7$. Next, we identified the statistically significant principal components and used the top 20 as input for t-Distributed Stochastic Neighbor Embedding dimensional reduction, using the TSNEPlot function. To identify cellular clusters, we adopted a graphbased clustering approach using FindClusters function with a 1.8 resolution. Finally, a multiclass SVM model (implementation from R package $\mathrm{bmrm}$ was trained on all cluster- assigned cells and genes were ranked according to their linear weights. For each class (i.e. clusters), genes with a significant linear weight (Z-test, FDR $\leq 0.05)$ were considered as enriched genes.

Pseudotime projection: APs, N1d and N4d cells at all embryonic ages identified in the cell clustering analysis were processed. Basal progenitors were not included in this analysis because N1d and N4d are overwhelmingly directly born from APs when using FT labeling (Telley et al. 2016; Govindan et al. 2018). The pseudotime alignment method performed was previously described (Mayer et al. 2018) and is summarized hereafter. In Fig. 2, Fig. 3 and figs. S5 and S6, we restricted the datasets to the high variable genes $(n=4,016)$ and performed dimensionality reduction using the prcomp function of $\mathrm{R}$ software. Taking into consideration the significant principal components (PCs) explaining at least 2\% of the total variance and using the $\mathrm{R}$ package princurve, we fitted a curve that described the maturation route (i.e. pseudo-birthdate or pseudo-differentiation) along which cells are organized. The beginning of the curve was established as the side where cell expressed the highest level of Sox2 (AP) for pseudodifferentiation or the highest level of Hmga2 (E12) for pseudo-birthdate. A maturation score reflecting the distance beginning of the curve-cell coordinate was attributed to each cell and normalized between 0 to 1 . We then restricted the dataset to the top 500 genes for each PCs and performed a "Partitioning Around Medoids" analysis using the PAM R package $(\mathrm{K}=6$, span $=0.6)$ to identify clusters of transcripts with similar expression dynamics along the pseudo-birthdate (Fig. 2, fig. S5) or pseudodifferentiation (Fig. 3, fig. S6). This approach was previously described elsewhere (Telley et al. 2016).

Ordinal regression models: We used an ordinal regression method to predict on one hand the birthdate and on the other hand the differentiation status of each cell. We restricted the analysis to the high variable genes $(n=4,016)$ defined earlier. As the cells are expected to be organized within a differentiation and a birthdate continuum, we used and adapted a previously described ordinal regression model (Teo et al. 2010) implemented in the bmrm R package. In our context, a single linear model is optimized to predict cell differentiation status irrespectively of the date of birth and conversely. The linear weight of the models is used to rank the genes according to their ability to predict each cell category and the best 100 genes in each model were considered. The ordinal regression models were then re-optimized on these subsets of genes. All reported predictions were obtained by 10 -fold cross-validation.

Transcriptional maps (Fig. 5): Cells were organized on a 2D grid based on their birthdate and differentiation status score. For this purpose, the data were linearly adjusted so that the average predicted values for each cardinal feature was aligned on to the relative knot of the grid. The gene expression at a given coordinate of the $2 \mathrm{D}$ space was further estimated as the average expression of its 15 nearest neighbors. All transcriptional landscapes of the most variable genes $(n=4,016)$ were further clustered by projecting genes onto a $2 \mathrm{D}$ t-SNE space and submitted to a k-means clustering $(\mathrm{K}=12)$.

\section{Electrophysiology}

Four hundred $\mu \mathrm{m}$-thick coronal slices were prepared from E12.5, E13.5, E14.5, E15.5 and E16.5 CD1 mice embryos and kept 30 minutes at $33^{\circ} \mathrm{C}$ in artificial cerebrospinal fluid (aCSF) containing $125 \mathrm{mM}$ 
$\mathrm{NaCl}, 2.5 \mathrm{mM} \mathrm{KCl}, 1 \mathrm{mM} \mathrm{MgCl} 2,2.5 \mathrm{mM} \mathrm{CaCl}_{2}, 1.25 \mathrm{mM} \mathrm{NaH}_{2} \mathrm{PO}_{4}, 26 \mathrm{mM} \mathrm{NaHCO}_{3}$ and $11 \mathrm{mM}$ glucose, saturated with $95 \% \mathrm{O}_{2}$ and $5 \% \mathrm{CO}_{2}$. Slices were then transferred in the recording chamber, submerged and continuously perfused with aCSF. The internal solution used for the experiments contained $140 \mathrm{mM}$ potassium methansulfonate, $2 \mathrm{mM} \mathrm{MgCl}$, $4 \mathrm{mM} \mathrm{NaCl} 0.2 \mathrm{mM}$ EGTA, $10 \mathrm{mM}$ HEPES, $3 \mathrm{mM} \mathrm{Na} 2 \mathrm{ATP}, 0.33 \mathrm{mM}$ GTP and $5 \mathrm{mM}$ creatine phosphate (pH 7.2, $295 \mathrm{mOsm}$ ). Cells in immediate proximity to the ventricular wall (i.e. putative APs) were patched and clamped at $-70 \mathrm{mV}$. A baseline stable holding current was first measured for 4 minutes, after which a 10-minute bath of $100 \mu \mathrm{M}$ of the glutamate transporter antagonist DL-TBOA (DL-threo- $\beta$-Benzyloxyaspartate) (Jabaudon et al. 1999) was applied and finally washed out. TBOA-induced currents were blocked by application of $25 \mu \mathrm{M}$ NBQX and $50 \mu \mathrm{M}$ D-APV (data not shown), consistent with activation of ionotropic glutamate receptors by increased extracellular levels of Glu (Jabaudon et al. 1999). Recorded currents were amplified (Multiclamp 700, Axon Instruments), filtered at $5 \mathrm{kHz}$, digitalized at $20 \mathrm{kHz}$ (National Instrument Board PCI-MIO-16E4, IGOR WaveMetrics), and stored on a personal computer for further analyses (IGOR PRO WaveMetrics). The net amplitude of TBOA induced currents was determined after subtraction of baseline holding current. Values are represented as mean $\pm \mathrm{SEM}$.

\section{$\underline{\text { Softwares }}$}

All single cell RNA sequencing analysis were perfomed using the R software with publicly available packages. GeneGo portal (https://portal.genego.com) was used to investigate the enriched gene ontology processes in Fig. 2 and Fig. 3 and the biomart R package served to extract the list of genes allocated to a defined ontology term. Cytoscape platform (Maere et al. 2005) associated with its plugin (Shannon et al. 2003) was used to construct the enrichment gene ontology processes network in supplementary fig. S9. For this purpose, the latest version of gene ontology (go-basic.obo) and gene association (gene_association.mgi) from the Gene Ontology Consortium website (www.geneontology.org) were given as input in Bingo. The string database (http://string-db.org) implemented in Cytoscape platform was used to determine the protein-protein interactions in figs S5, S6 and S7.

\section{$\underline{\text { References }}$}

Govindan S. et al., 2018. In vivo pulse labeling of isochronic cohorts of cells in the central nervous system using FlashTag. Nature Protocols. DOI : 10.1038/s41596-018-0038-1.

Jabaudon, D. et al., 1999. Inhibition of uptake unmasks rapid extracellular turnover of glutamate of nonvesicular origin. Proceedings of the National Academy of Sciences of the United States of America, 96(15), pp.87338738 .

Maere, S., Heymans, K. \& Kuiper, M., 2005. BiNGO: a Cytoscape plugin to assess overrepresentation of gene ontology categories in biological networks. Bioinformatics (Oxford, England), 21(16), pp.3448-3449.

Mayer, C. et al., 2018. Developmental diversification of cortical inhibitory interneurons. Nature, 555(7697), pp.457-462.

Shannon, P. et al., 2003. Cytoscape: a software environment for integrated models of biomolecular interaction networks. Genome research, 13(11), pp.2498-2504.

Telley, L. et al., 2016. Sequential transcriptional waves direct the differentiation of newborn neurons in the mouse neocortex. Science (New York, NY), 351(6280), pp.1443-1446.

Teo, C.H. et al., 2010. Bundle Methods for Regularized Risk Minimization. Journal of Machine Learning Research, 11(Jan), pp.311-365. 
A

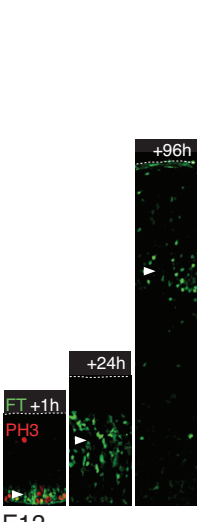

E12

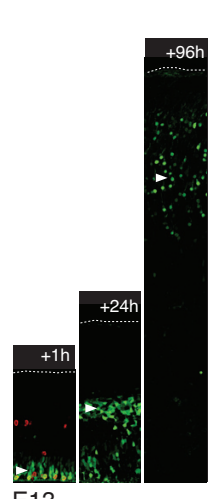

E13

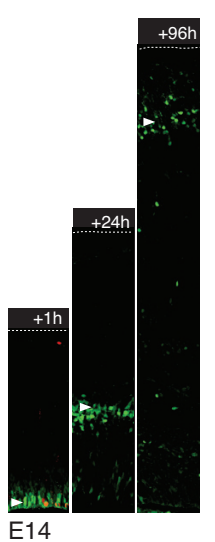

E14

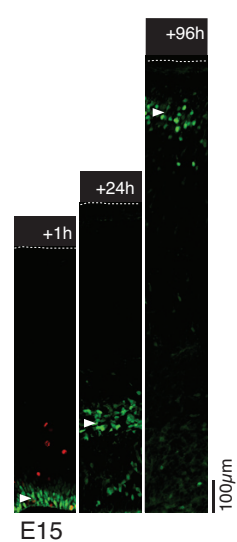

E15
B

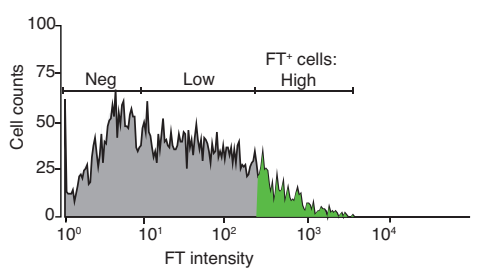

C

Fluidigm $\mathrm{C} 1$

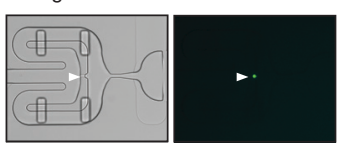

D
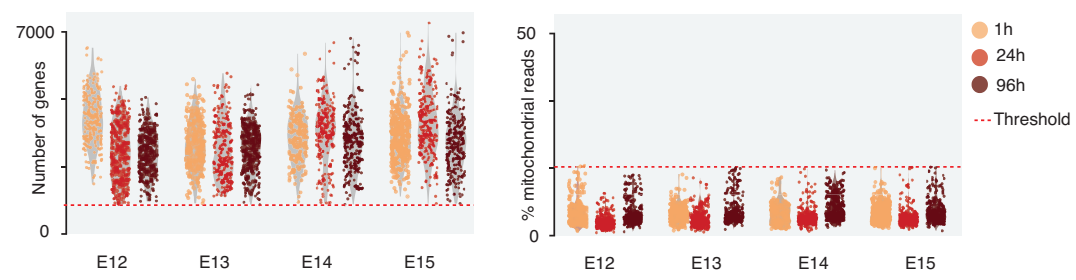

Fig. S1. scRNA sequencing of isochronic cohorts of cells in the developing neocortex. (A) FT injection labels isochronic cohorts of cortical cells which were collected either 1, 24 or 96 hours after labeling. E12 illustration modified from Fig.1. (B) FT+ cells were FAC-sorted and (C) captured on a $\mathrm{C} 1$ microfluidic device for scRNAseq processing. (D) Violin plots showing the number of genes and the percentage of mitochondrial genes detected in each single cell. Lower $(<1000$ genes $)$ and upper $(>$ $17 \%$ mitochondrial genes) accepted thresholds are displayed. 
A

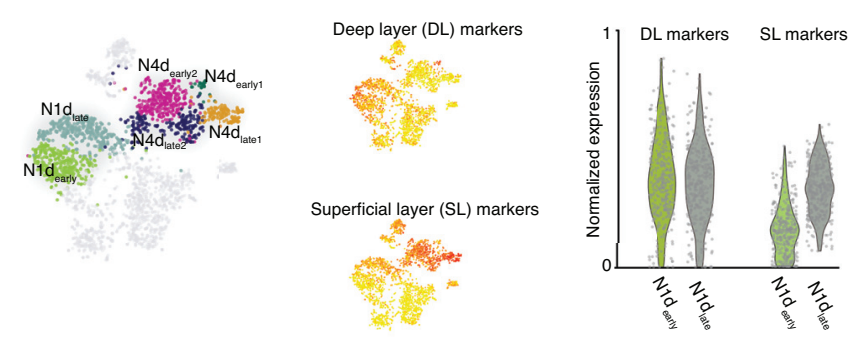

Deep layer markers
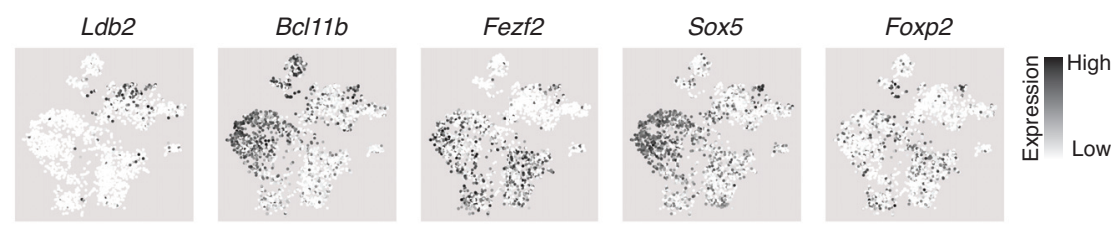

Superficial layer markers
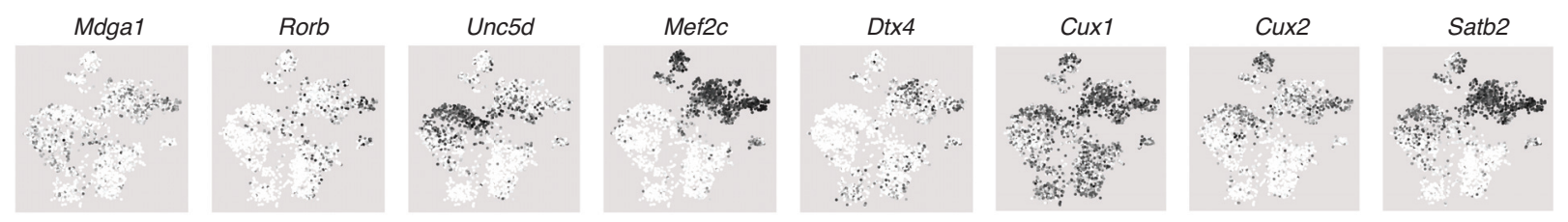

B

Astrocytes
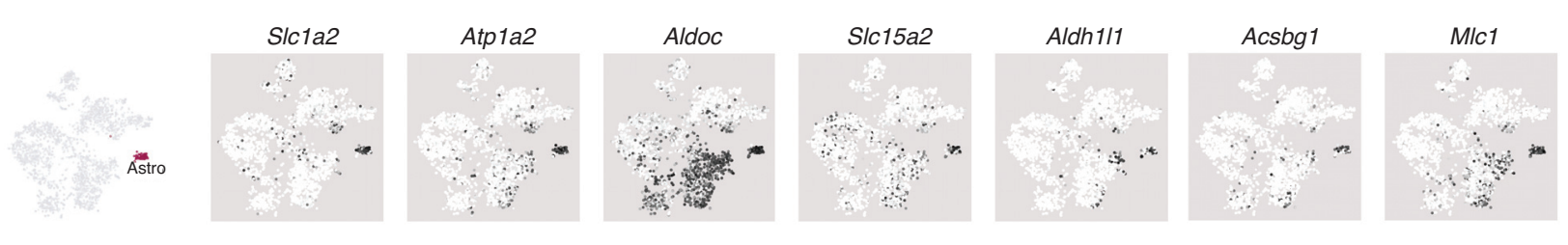

C

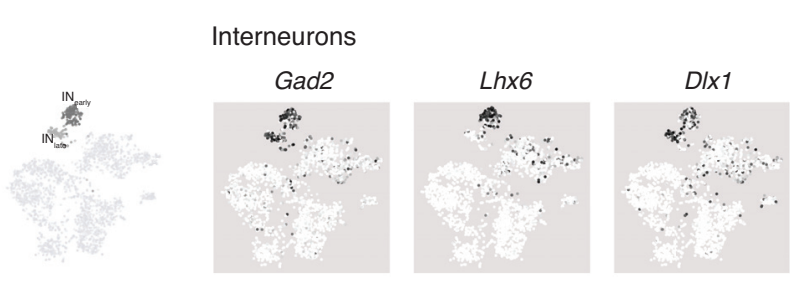

Fig. S2. Expression of select genes in neurons, astrocytes, and interneurons. (A) Feature plots showing expression of classical deep layer (DL) and superficial layer (SL) markers in 1-day and 4-day-old neurons across corticogenesis. Note that N1dlate neurons (i.e. E14 and E15-born neurons) initially and transiently express DL markers. (C) Expression of select astrocytes markers. (D) Expression of select ventral pallial-derived interneuron markers. 

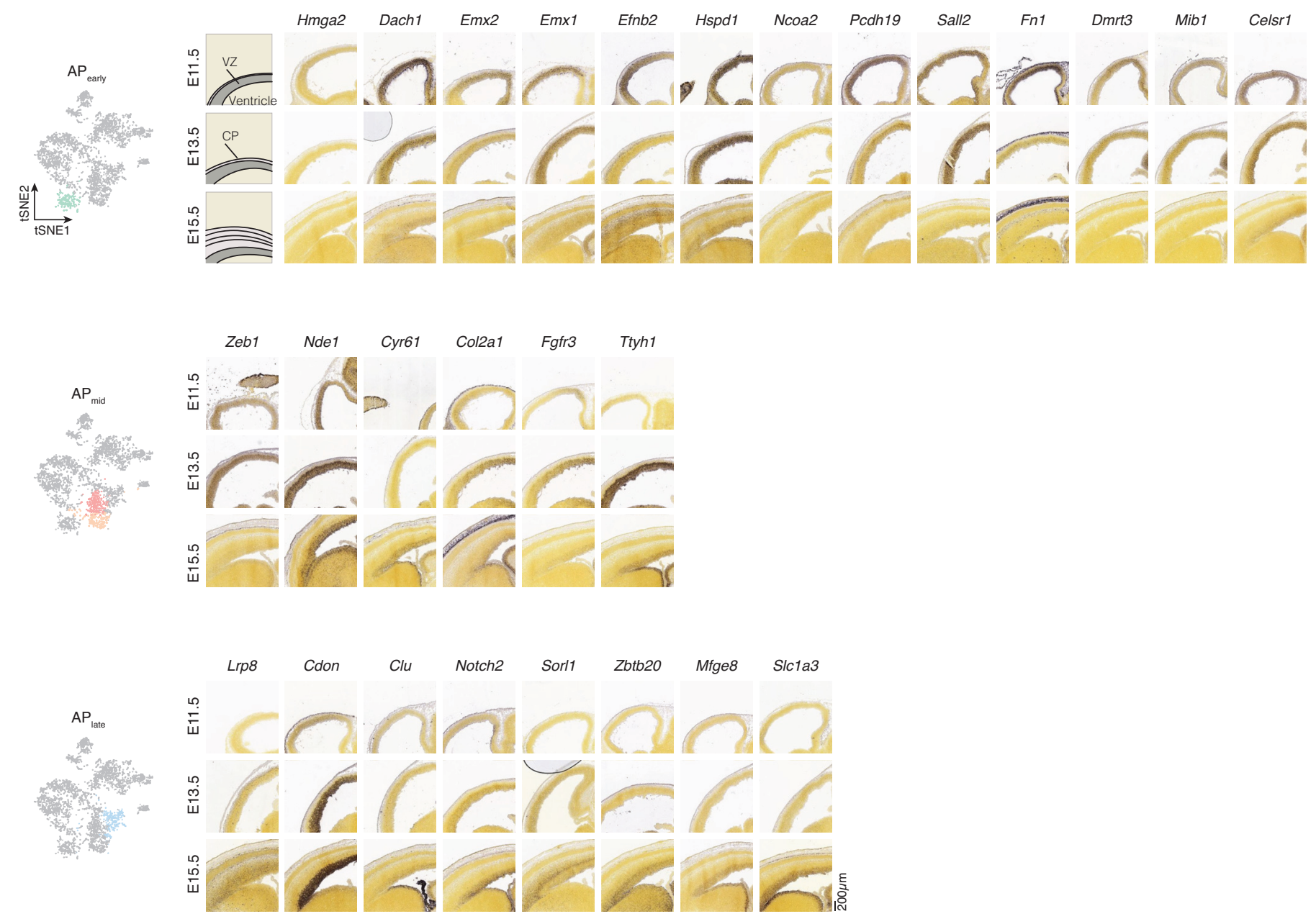

Fig. S3. Spatio-temporal expression of the most enriched genes in AP clusters. In situ hybridization of genes enriched in APearly, APmid, and APlate clusters. The ISH merged layouts are also presented in Fig. 1E. Source of ISH: Allen Developing Mouse Brain Atlas. 

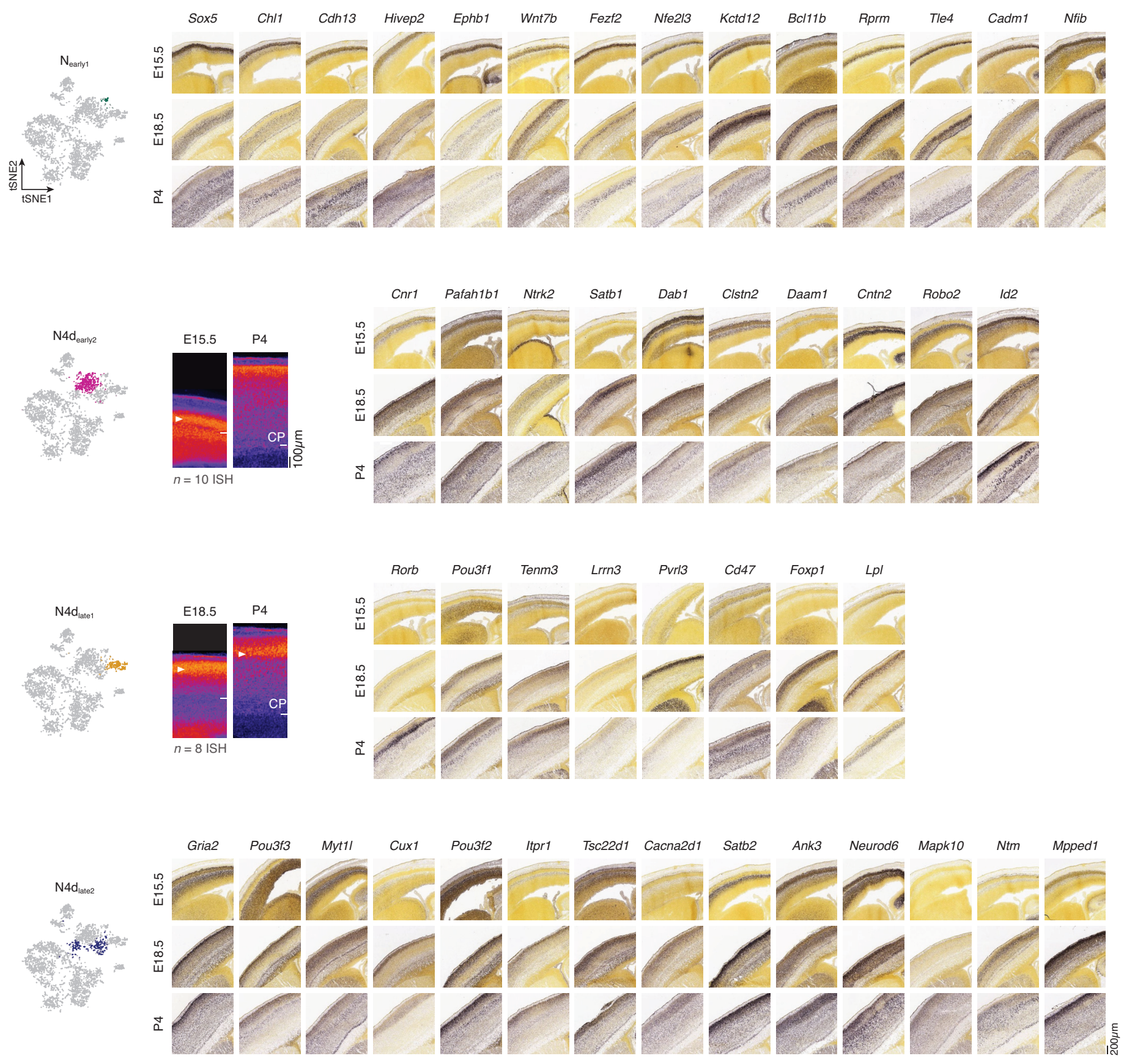

Fig. S4. Spatio-temporal expression of the most enriched genes in 4-day-old clusters. Individual and merged in situ hybridization of genes enriched in N4dearly1, N4dearly2, N4dlate1 and N4dlate2 clusters. The ISH merged layouts for N4dearly1 and N4dlate2 are also presented in Fig. 1E. Source of ISH: Allen Developing Mouse Brain Atlas. 
A
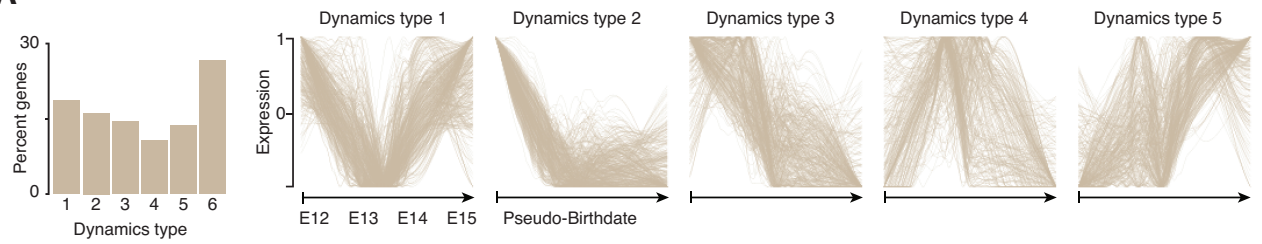

Dynamics type 6

B
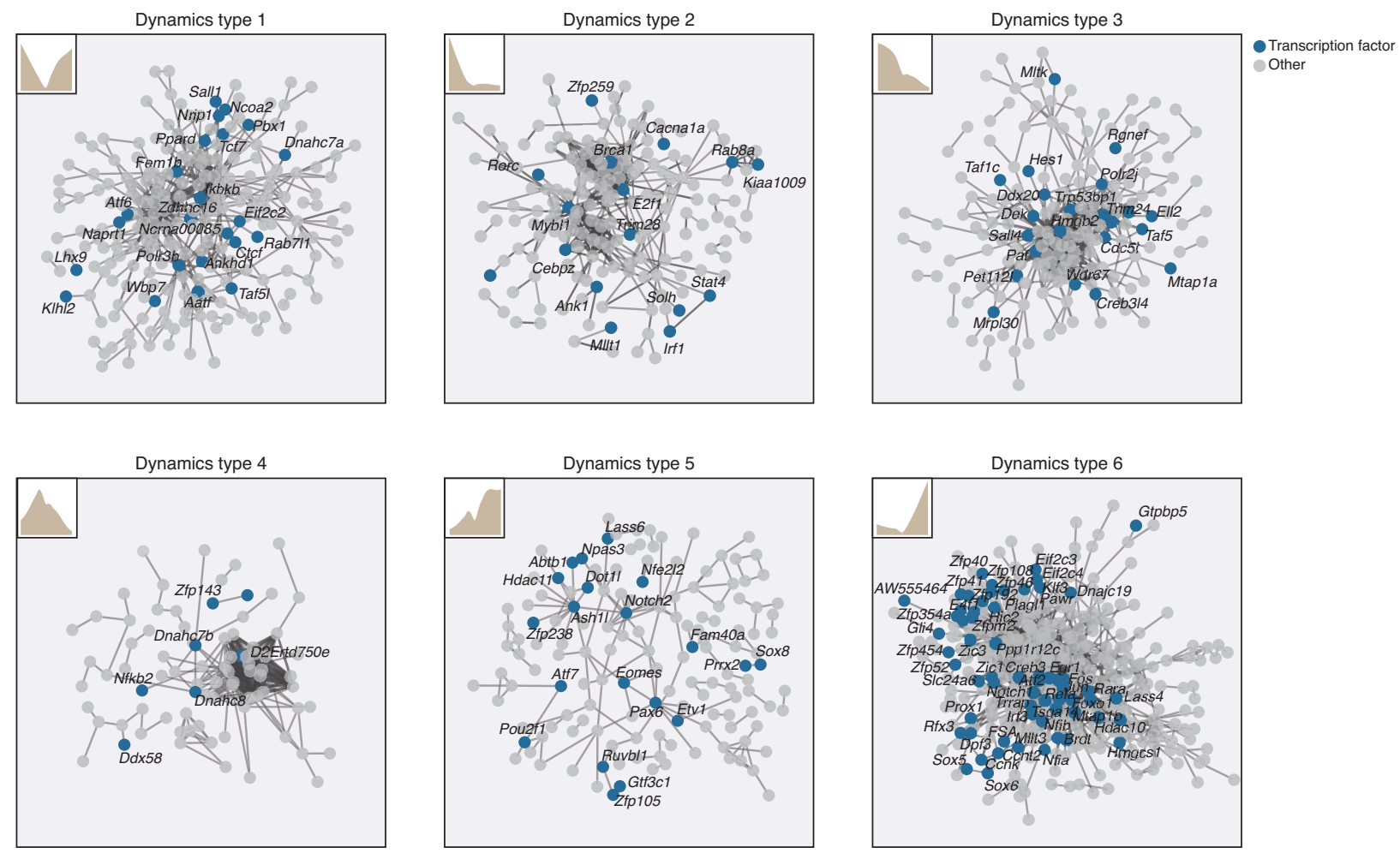

C
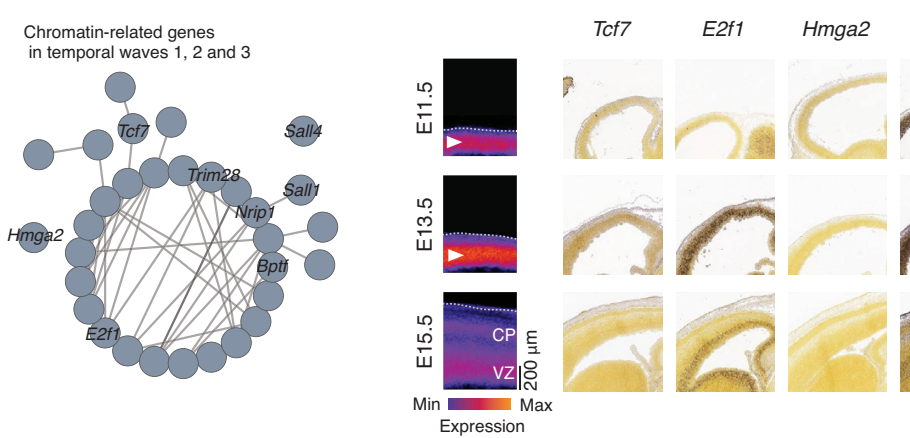

Trim28

Nrip1

Sall4

Bptf Sall1

sis

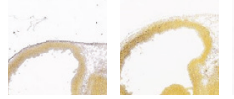

C.

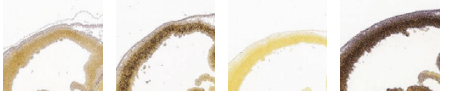

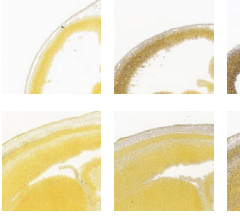
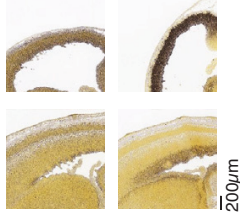

Fig. S5. Sequential AP transcriptional states across corticogenesis. (A) Genes distribution within the six sequential AP transcriptional states. (B) Global protein-protein interactome for each AP state, from https://string-db.org/. Unassigned genes are not displayed. (C) Interactome of chromatin-related genes in AP early states. (D) In situ hybridization of chromatin-related genes at early AP states. Source of ISH: Allen Developing Mouse Brain Atlas. 
A

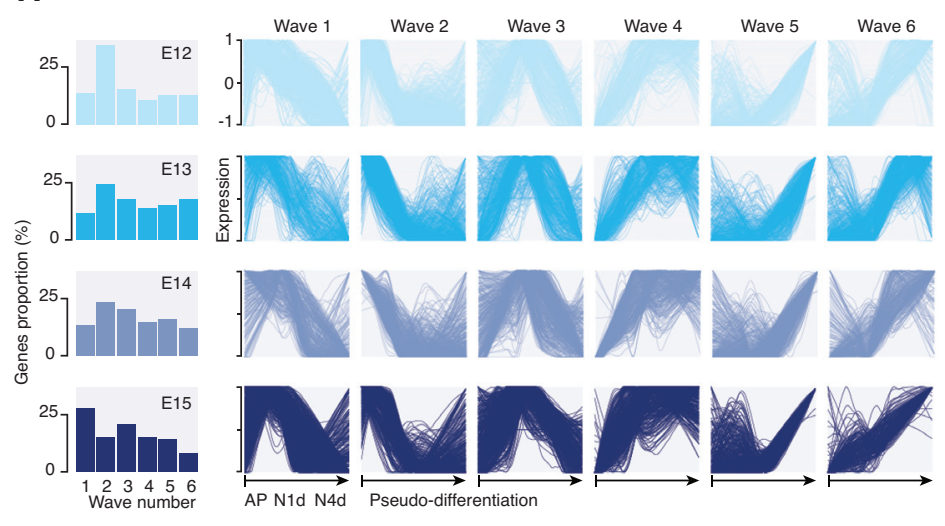

C
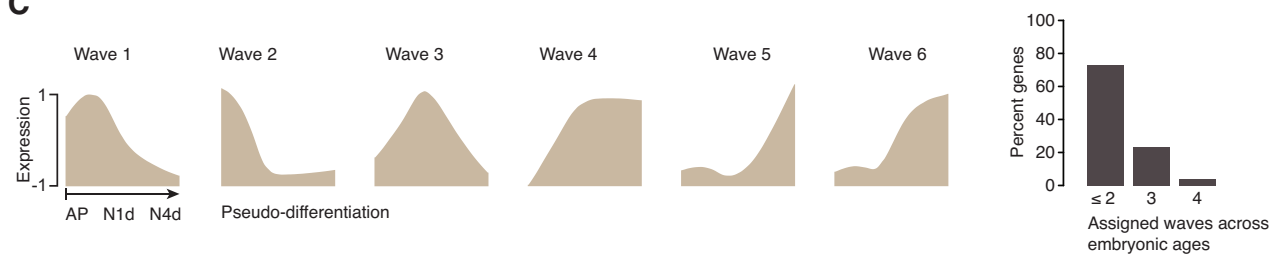

mbryonic ages

D

Genes distributed within $\leq 2$ waves accross embryonic ages
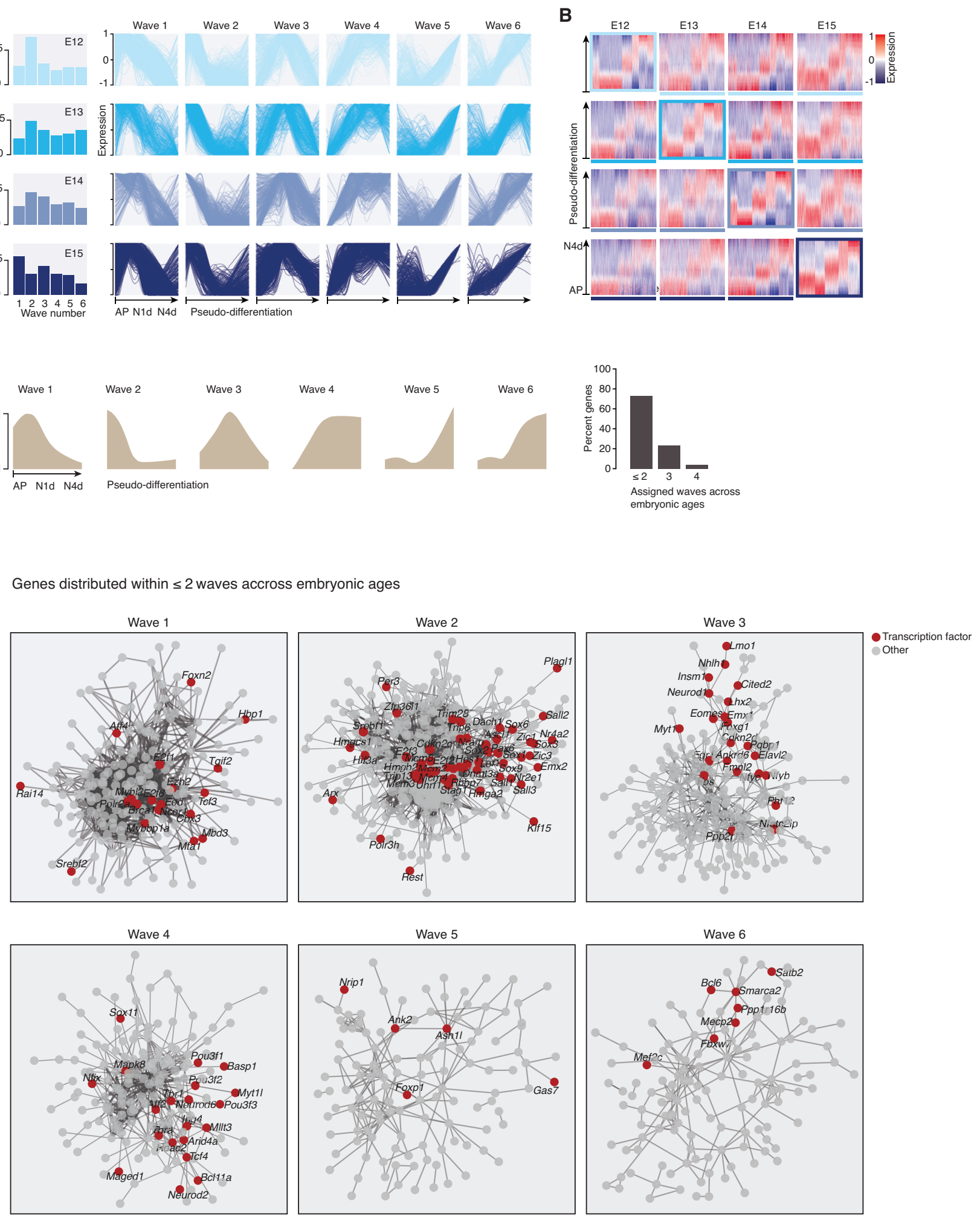
A
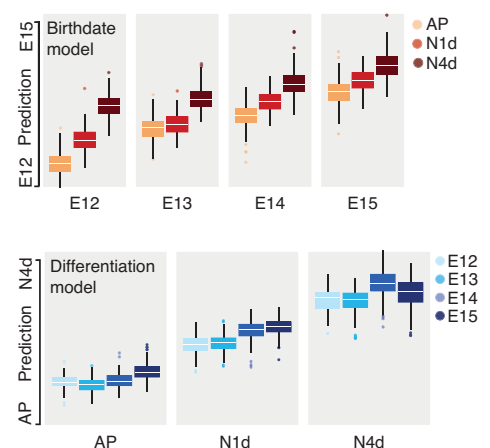

C

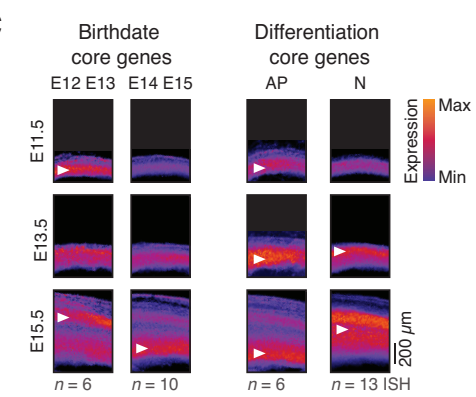

B

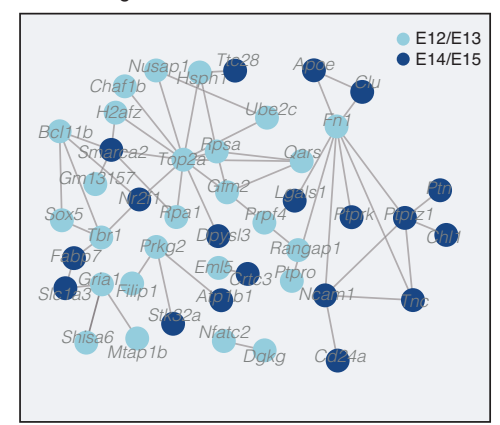

Differentiation genes

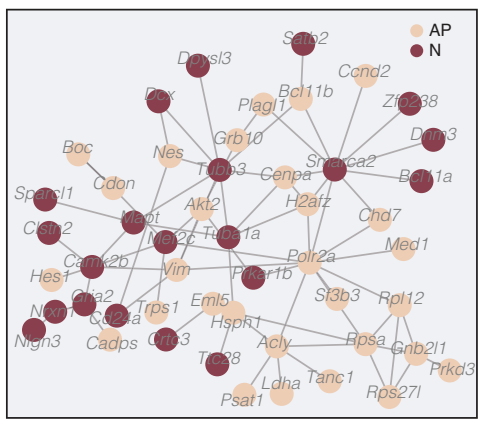

D
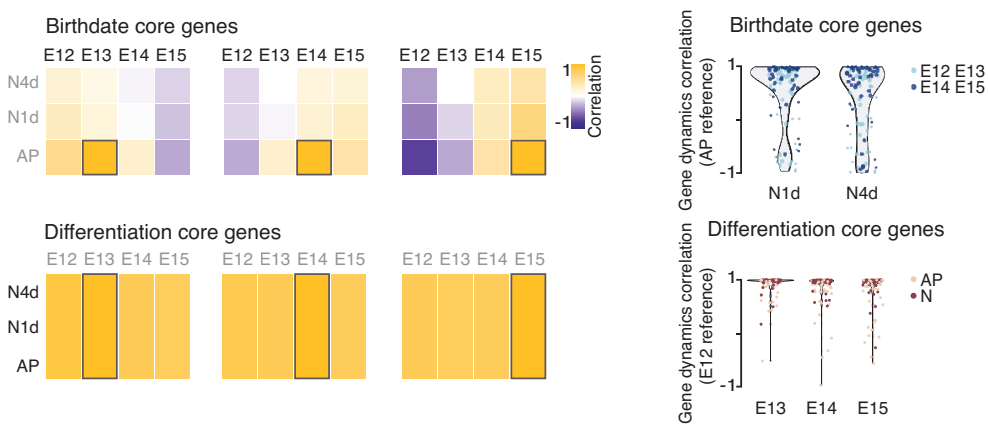

E

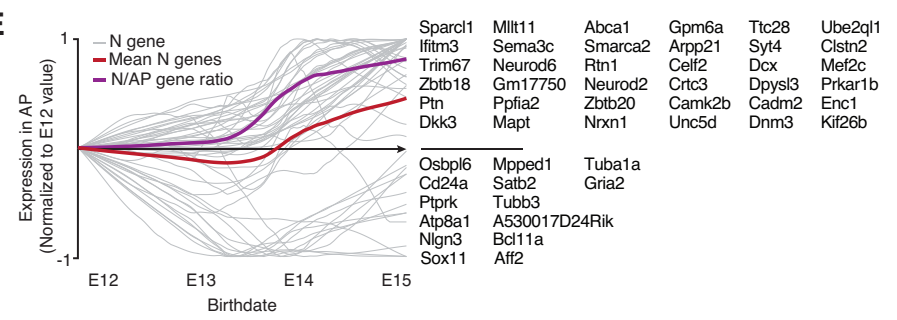

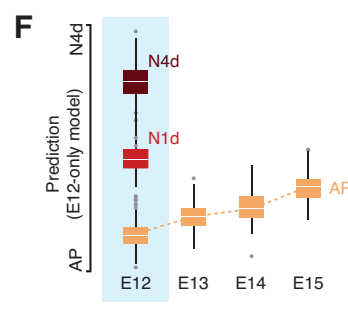

Fig. S7. 2D modelization of corticogenesis. (A) Birthdate and differentiation scores obtained from the two models for each condition. (B) Analysis of protein-protein interactions using the STRING database (http://string-db.org) suggests that gene products interact based on their temporal dynamics (left) or cellular specificity (right). Unassigned genes are not displayed. (C) Overlay of ISH from the Allen Developing Mouse Brain Atlas (www.brain-map.org) confirming the proper spatio-temporal dynamics of select core genes. Early genes: Hes1, Hmga2, Tbr1, Fn1, Nfatc2, Sox5. Late genes: Nrxn1, Cttnbp2, Clu, Nr2f1, Lgals1, Bcan, Tnc, Unc5d, Slcla3, Mfge8. AP genes: Cdon, Hes1, Plagl1, Nes, Hmga2, Arx. N genes: Trps1, Unc5d, Sox11, Nrxn, Cd24a, Mpped1, Bcl11a, Neurod6, Satb2, Dcx, Mapt, Gria2, Tubb3. (D) Top: Birthdate-associated core genes are temporally dynamic and daughter cells acquire embryonic stage-specific transcriptional birthmarks. Bottom: In contrast, differentiation status-associated core genes are conserved across corticogenesis. Boxed area represents value of reference for correlation. Right: Correlations in gene expression dynamics stratified for early (E12, E13) and late (E14, E15) embryonic ages. (E) Expression of the core neuronal genes $(\mathrm{n}=50)$ within APs increases with embryonic age. (F) E12-15 APs progressively become "neuralized". Differentiation model build exclusively with E12 data as a training dataset; E13-E15 APs are classified as progressively more neuron-like using this model. 

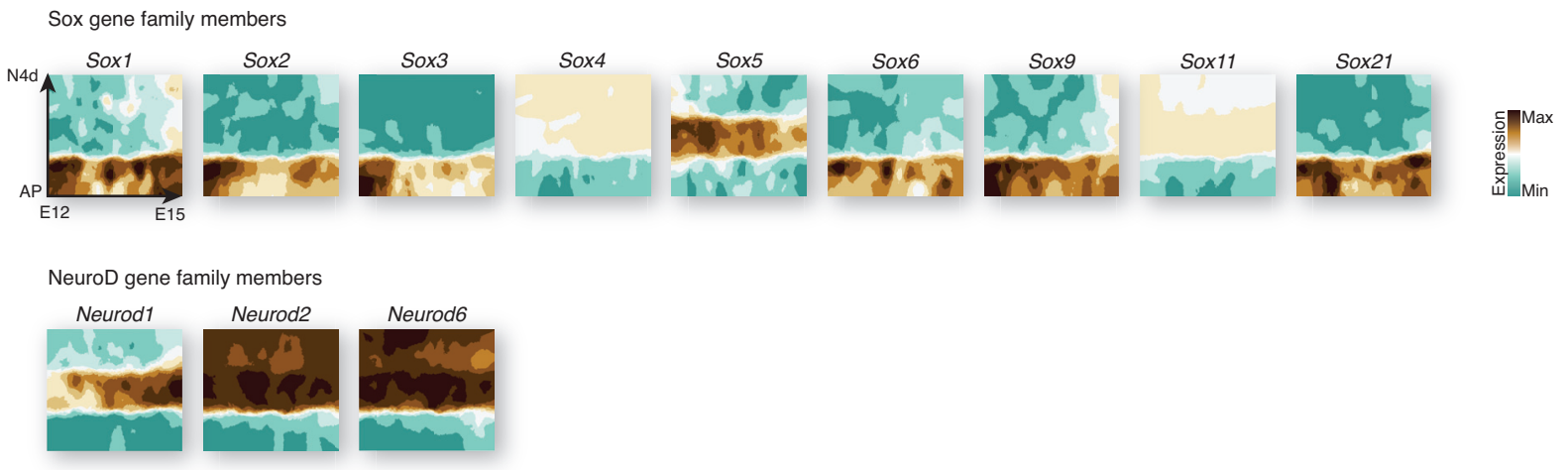

\section{Trim gene family members}
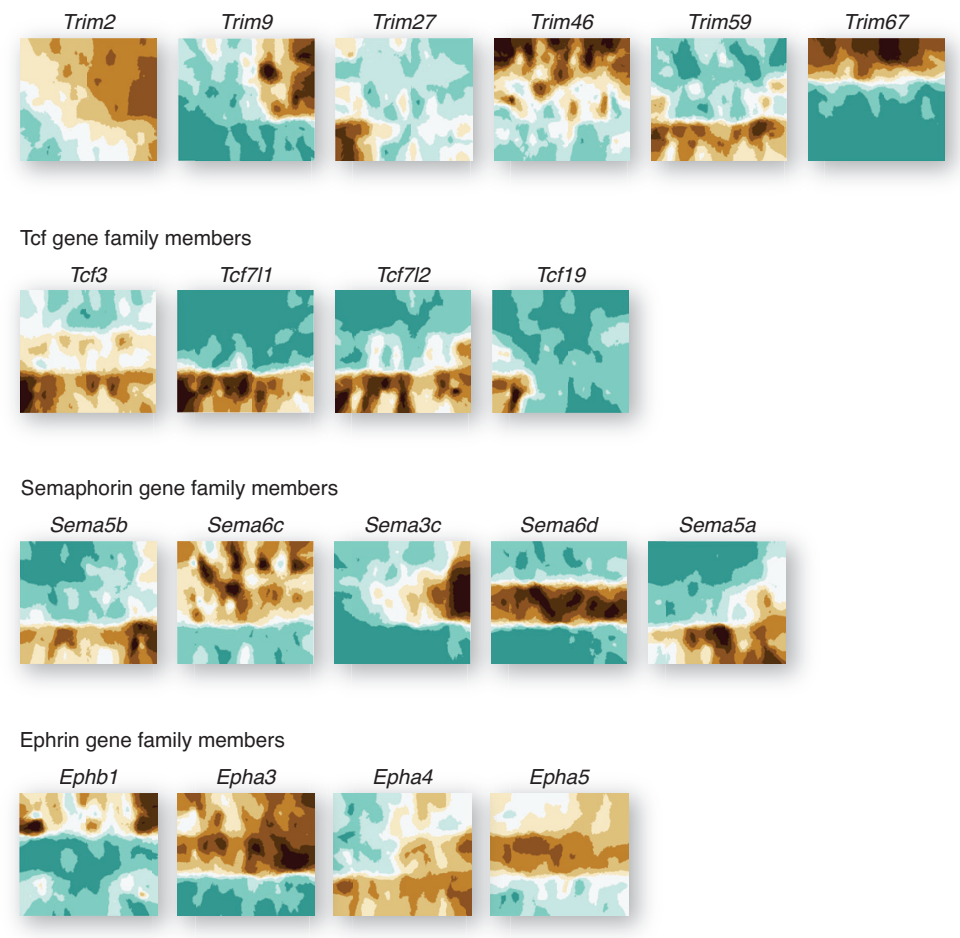

Fig. S8. Examples of members of gene families and their associated transcriptional maps. Only the genes with the most sharply delineated expression patterns are shown for Semaphorins and Ephrins. 

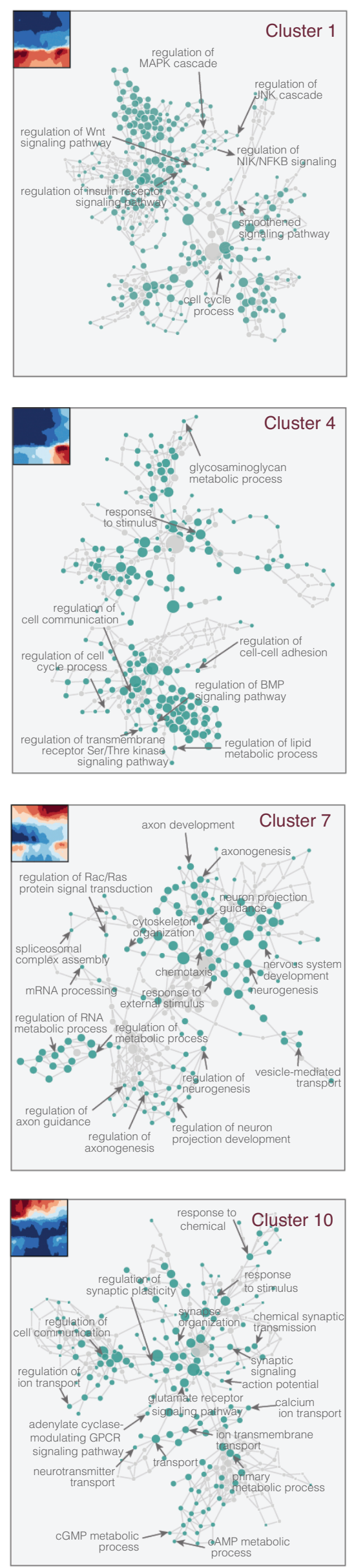
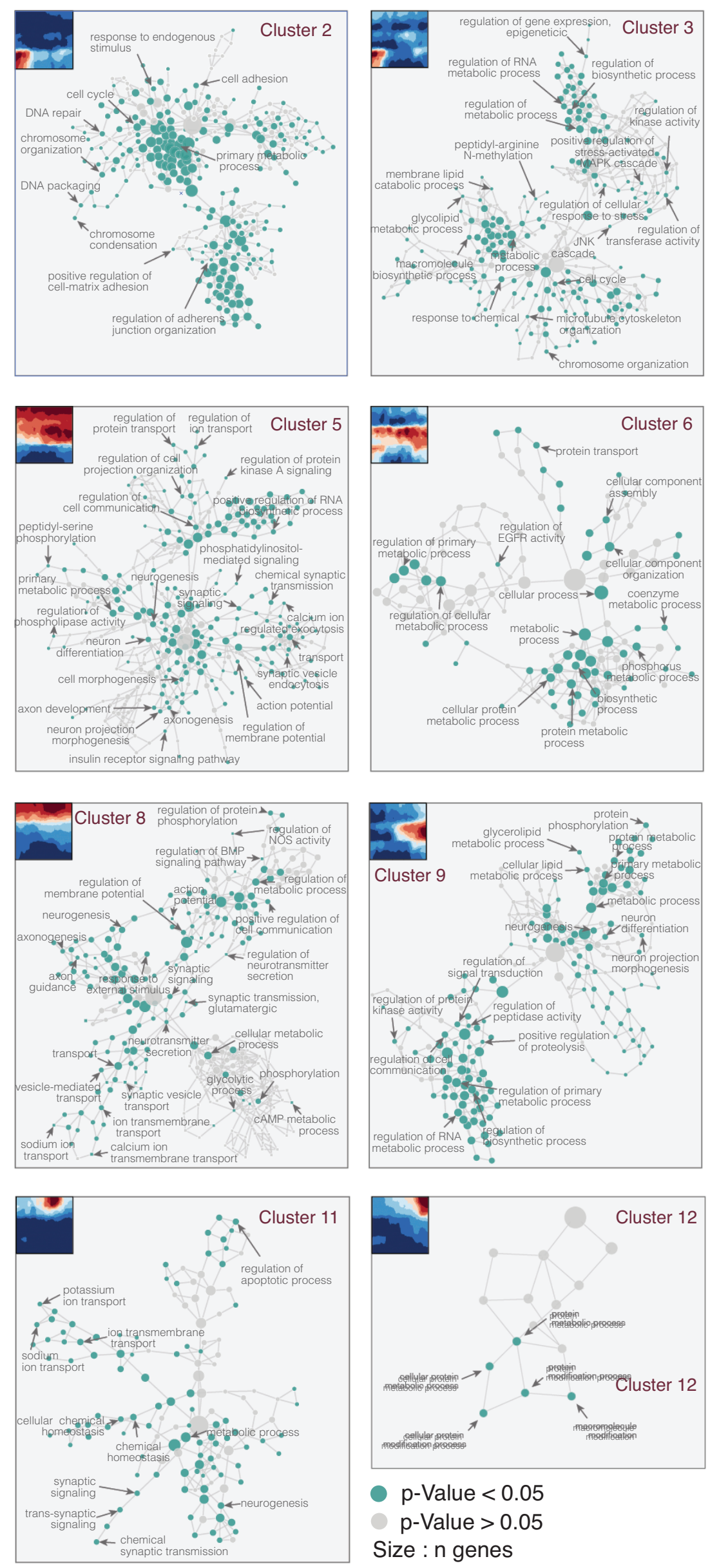

p-Value $<0.05$

$p$-Value $>0.05$

Size : n genes

Fig. S9. Cluster-based gene ontology networks. Display of ontological hierarchies for individual clusters highlights cluster-specific biological processes. 